\title{
Cyclic sieving and rational Catalan theory
}

\author{
Michelle Bodnar Brendon Rhoades \\ Department of Mathematics \\ University of California, San Diego \\ La Jolla, CA, 92093-0112, USA \\ \{mbodnar, bprhoades\}@math.ucsd.edu
}

Submitted: Nov 6, 2015; Accepted: Mar 16, 2016; Published: Apr 1, 2016

Mathematics Subject Classifications: 05A18, 05A19, 05E18

\begin{abstract}
Let $a<b$ be coprime positive integers. Armstrong, Rhoades, and Williams (2013) defined a set NC $(a, b)$ of 'rational noncrossing partitions', which form a subset of the ordinary noncrossing partitions of $\{1,2, \ldots, b-1\}$. Confirming a conjecture of Armstrong et. al., we prove that $\mathrm{NC}(a, b)$ is closed under rotation and prove an instance of the cyclic sieving phenomenon for this rotational action. We also define a rational generalization of the $\mathfrak{S}_{a}$-noncrossing parking functions of Armstrong, Reiner, and Rhoades (2015).
\end{abstract}

Keywords: cyclic sieving, noncrossing partition, rational Catalan theory, parking function

\section{Introduction}

This paper is about generalized noncrossing partitions arising in rational Catalan combinatorics. A set partition of $[n]:=\{1,2, \ldots, n\}$ is noncrossing if its blocks do not cross when drawn on a disk whose boundary is labeled clockwise with $1,2, \ldots, n$. Noncrossing partitions play a key role in algebraic and geometric combinatorics. Along with an ever-expanding family of other combinatorial objects, the noncrossing partitions of $[n]$ are famously counted by the Catalan number

$$
\operatorname{Cat}(n)=\frac{1}{n+1}\left(\begin{array}{c}
2 n \\
n
\end{array}\right)=\frac{1}{2 n+1}\left(\begin{array}{c}
2 n+1 \\
n
\end{array}\right) .
$$

Given a Fuss parameter $m \geqslant 1$, the Fuss-Catalan number is

$$
\mathrm{Cat}^{(m)}(n)=\frac{1}{m n+n+1}\left(\begin{array}{c}
m n+n+1 \\
n
\end{array}\right) .
$$


When $m=1$, this reduces to the classical Catalan number. Many Catalan objects have natural Fuss-Catalan generalizations. In the case of noncrossing partitions, the FussCatalan number $\mathrm{Cat}^{(m)}(n)$ counts noncrossing set partitions of $[m n]$ which are $m$-divisible in the sense that every block has size divisible by $m$.

For coprime positive integers $a$ and $b$, the rational Catalan number is Cat $(a, b)=$ $\frac{1}{a+b}\left(\begin{array}{c}a+b \\ a, b\end{array}\right)$. Observe that $\operatorname{Cat}(n, n+1)=\operatorname{Cat}(n)$ and $\operatorname{Cat}(n, m n+1)=\operatorname{Cat}^{(m)}(n)$, so that rational Catalan numbers are a further generalization of Fuss-Catalan numbers. Inspired by favorable representation theoretic properties of the rational Cherednik algebra attached to the symmetric group $\mathfrak{S}_{a}$ at parameter $\frac{b}{a}$, the research program of rational Catalan combinatorics seeks to further generalize Catalan combinatorics to the rational setting.

Some rational generalizations of Catalan objects have been around for decades - the rational analog of a Dyck path dates back at least to the probability literature of the 1940s. Armstrong, Rhoades, and Williams used rational Dyck paths to define rational analogs of polygon triangulations, noncrossing perfect matchings, and noncrossing partitions [4]. This paper goes deeper into the study of rational noncrossing partitions.

For coprime parameters $a<b$, Armstrong et. al. defined the $a, b$-noncrossing partitions to be a subset $\mathrm{NC}(a, b)$ of the collection of noncrossing partitions of $[b-1]$ arising from a laser construction involving rational Dyck paths (see Section 2 for details). It was shown that $\mathrm{NC}(a, b)$ is counted by Cat $(a, b)$, as it should be, and that $\mathrm{NC}(n, m n+1)$ is the set of $m$-divisible noncrossing partitions of $[m n]$, as it should be.

However, the construction of $\mathrm{NC}(a, b)$ in [4] was indirect and involved the intermediate object of rational Dyck paths. This left open the question of whether many of the fundamental properties of classical noncrossing partitions generalize to the rational case. For example, it was unknown whether the set $\mathrm{NC}(a, b)$ is closed under the dihedral group of symmetries of the disk with $b-1$ labeled boundary points. Consequently, the rich theory of counting noncrossing set partitions fixed by a dihedral symmetry (see [10]) lacked a rational extension. Moreover, the unknown status of rotational closure made it difficult to generalize the noncrossing parking functions of Armstrong, Reiner, and Rhoades [3] (or the 2-noncrossing partitions of Edelman [7]) to the rational setting. The core problem was that the natural dihedral symmetries of noncrossing partitions are harder to visualize on the level of Dyck paths, even in the classical case.

The purpose of this paper is to resolve the issues in the last paragraph to support $\mathrm{NC}(a, b)$ as the 'correct' definition of the rational noncrossing partitions. We will prove the following.

- $\mathrm{NC}(a, b)$ is closed under dihedral symmetries (Corollary 3.16).

- The action of rotation on NC $(a, b)$ exhibits a cyclic sieving phenomenon generalizing that for the action of rotation on classical noncrossing partitions (Theorem 5.3).

- The numerology of partitions in $\mathrm{NC}(a, b)$ with a nontrivial rotational symmetry generalizes that of classical noncrossing partitions with a nontrivial rotational symmetry (Corollaries 4.10, 4.11, 4.12). 
- Partitions in $\mathrm{NC}(a, b)$ can be decorated to obtain a $\mathfrak{S}_{a} \times \mathbb{Z}_{b-1}$-set of rational noncrossing parking functions $\operatorname{Park}^{N C}(a, b)$. The formula for the permutation character of this set generalizes the corresponding formula for the classical case (Theorem 6.3).

The key to obtaining the rational extensions of classical results presented above will be to develop a better understanding of the set $\mathrm{NC}(a, b)$. We will give two new characterizations of this set (Propositions 3.3 and 3.15). The more important of these will involve an idea genuinely new to rational Catalan combinatorics: a new measure of size for blocks of set partitions in $\mathrm{NC}(a, b)$ called rank.

In the Fuss-Catalan case $(a, b)=(n, m n+1)$, the rank of a block is determined by its cardinality (Proposition 3.6). However, rank and cardinality diverge at the rational level of generality. The main heuristic of this paper is that:

The rank of a block of a rational noncrossing partition is a better measure of its size than its cardinality.

In Section 3, we will prove that rank shares additivity and dihedral invariance properties with size. We will prove that ranks (unlike cardinalities) characterize which noncrossing set partitions of $[b-1]$ lie in $\mathrm{NC}(a, b)$. In Section 6 , ranks (not cardinalities) will be used to define and study rational noncrossing parking functions. In Sections 4 and 5, ranks (not cardinalities) will be used to give rational analogs of enumerative results for noncrossing partitions with rotational symmetry.

\section{Background}

\subsection{Rational Dyck paths}

The prototypical object in rational Catalan combinatorics is the rational Dyck path. Let $(a, b)$ be coprime positive integers. An $a, b$-Dyck path (or just a Dyck path when $a$ and $b$ are clear from context) is a lattice path in $\mathbb{Z}^{2}$ consisting of north and east steps which starts at $(0,0)$ ends at $(b, a)$, and stays above the line $y=\frac{a}{b} x$. The 5,8-Dyck path $N E N E E N N E N E E E$ is shown in Figure 1. When $(a, b)=(n, n+1)$, rational Dyck paths are equivalent to classical Dyck paths - lattice paths from $(0,0)$ to $(n, n)$ which stay weakly above $y=x$.

If $D$ is an $a, b$-Dyck path, a valley of $D$ is a lattice point $P$ on $D$ such that $P$ is immediately preceded by an east step and succeeded by a north step. A vertical run of $D$ is a maximal contiguous sequence of north steps; the number of vertical runs equals the number of valleys plus one. The 5,8-Dyck path shown in Figure 1 has 4 valleys. The vertical runs of this path have sizes $1,1,2$, and 1 .

The numerology associated to rational Dyck paths generalizes that of classical Dyck

paths. The number of $a, b$-Dyck paths is the rational Catalan number Cat $(a, b)=\frac{1}{a+b}\left(\begin{array}{c}a+b \\ a, b\end{array}\right)$. The set of $a, b$-Dyck paths $D$ with $k$ vertical runs is counted by the rational Narayana number

$$
\operatorname{Nar}(a, b ; k):=\frac{1}{a}\left(\begin{array}{l}
a \\
k
\end{array}\right)\left(\begin{array}{l}
b-1 \\
k-1
\end{array}\right) .
$$




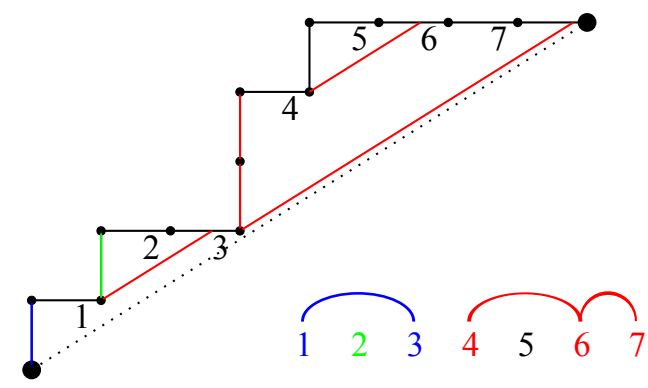

Figure 1: A 5, 8-Dyck path and the corresponding 5, 8-noncrossing partition. The visibility bijection is shown with colors.

Given a length $a$ vector of nonnegative integers $\mathbf{r}=\left(r_{1}, r_{2}, \ldots, r_{a}\right)$ satisfying $\sum i r_{i}=a$, the number of $a, b$-Dyck paths with $r_{i}$ vertical runs of size $i$ for $1 \leqslant i \leqslant a$ is given by the rational Kreweras number

$$
\operatorname{Krew}(a, b ; \mathbf{r}):=\frac{1}{b}\left(\begin{array}{c}
b \\
r_{1}, r_{2}, \ldots, r_{a}, b-k
\end{array}\right)=\frac{(b-1) !}{r_{1} ! r_{2} ! \cdots r_{a} !(b-k) !},
$$

where $k=\sum r_{i}$ is the total number of vertical runs. For example, the 5,7-Dyck path shown in Figure 2 contributes to $\operatorname{Krew}(5,7 ; \mathbf{r})$, where $\mathbf{r}=(1,2,0,0,0)$.

\subsection{Noncrossing partitions}

A set partition $\pi$ of $[n]$ is called noncrossing if, for all indices $1 \leqslant i<j<k<\ell \leqslant n$, we have that $i \sim k$ in $\pi$ and $j \sim \ell \in \pi$ together imply that $i \sim j \sim k \sim \ell$ in $\pi$. Equivalently, the set partition $\pi$ is noncrossing if and only if the convex hulls of the blocks of $\pi$ do not intersect when drawn on the disk with boundary points labeled clockwise with $1,2, \ldots, n$.

We let $\mathrm{NC}(n)$ denote the collection of noncrossing partitions of $[n]$. The rotation operator rot acts on the index set $[n]$, the power set $2^{[n]}$, and the collection $\mathrm{NC}(n)$ by the permutation $\left(\begin{array}{ccccc}1 & 2 & \ldots & n-1 & n \\ 2 & 3 & \ldots & n & 1\end{array}\right)$. These three sets also carry an action of the reflection operator $\mathrm{rfn}$ by the permutation $\left(\begin{array}{ccccc}1 & 2 & \ldots & n-1 & n \\ n & n-1 & \ldots & 2 & 1\end{array}\right)$. Together, rot and $r f n$ generate a dihedral action on these sets.

\subsection{Rational noncrossing partitions}

In [4], rational Dyck paths were used to construct a rational generalization of the noncrossing partitions. Let $D$ be an $a, b$-Dyck path and let $P \neq(0,0)$ be a lattice point which is at the bottom of a north step of $D$. The laser $\ell(P)$ is the line segment of slope $\frac{a}{b}$ which is 'fired' northeast from $P$ and continues until it intersects the Dyck path $D$. By coprimality, the east endpoint of $\ell(P)$ is necessarily on the interior of an east step of $D$.

Let $D$ be an $a, b$-Dyck path. We define a set partition $\pi(D)$ of $[b-1]$ as follows. Label the east ends of the non-terminal east steps of $D$ from left to right with $1,2, \ldots, b-1$ and 
fire lasers from all of the valleys of $D$. The set partition $\pi(D)$ is defined by the 'visibility' relation

$i \sim j$ if and only if the labels $i$ and $j$ are not separated by laser fire.

(Here we consider labels to lie slightly below their lattice points.) By construction, the set partition $\pi(D)$ is noncrossing.

An example of this construction when $(a, b)=(5,8)$ is shown in Figure 1. If $D$ is an $a, b$-Dyck path, we have a natural 'visibility' bijection from the set of vertical runs of $D$ to the set of blocks of $\pi(D)$ which associates a vertical run at $y=i$ to the block of $\pi(D)$ whose minimum element is $i+1$. The visibility bijection is shown with colors in Figure 1 .

It will be convenient to think of the lasers fired from the valleys of a Dyck path $D$ in terms of their endpoints. We let the laser set $\ell(D)$ of the $a, b$-Dyck path $D$ be the set of pairs $(i, j)$ such that $D$ contains a laser starting from a valley with $x$-coordinate $i$ and ending in the interior of an east step with west $x$-coordinate $j$. For the 5,8 -Dyck path $D$ shown in Figure 1, we have

$$
\ell(D)=\{(1,2),(3,7),(4,5)\} .
$$

For $a<b$ coprime, we define the set of admissible lasers

$$
A(a, b):=\left\{(i, j): 1 \leqslant i<j \leqslant b-1 \text { and } j-i=\left\lfloor\frac{r b}{a}\right\rfloor \text { for some } r=1,2, \ldots, a-1\right\} .
$$

Slope considerations show that $(i, j) \in \ell(D)$ for some $a, b$-Dyck path $D$ if and only if $(i, j) \in A(a, b)$. By considering $\pi(D)$ for all possible $a, b$-Dyck paths $D$, we get the set of $a, b$-noncrossing partitions

$$
\mathrm{NC}(a, b):=\{\pi(D): D \text { an } a, b \text {-Dyck path }\}
$$

(This is called the set of inhomogeneous a,b-noncrossing partitions in [4].) It is clear from construction that $\mathrm{NC}(a, b) \subseteq \mathrm{NC}(b-1)$. Some basic facts about $a, b$-noncrossing partitions are as follows.

Proposition 2.1. Let $a<b$ be coprime positive integers.

1. The map

$$
\pi:\{a, b-D y c k \text { paths }\} \rightarrow \mathrm{NC}(a, b)
$$

is bijective, so that $|\mathrm{NC}(a, b)|=\operatorname{Cat}(a, b)$ and the number of $a, b$-noncrossing partitions with $k$ blocks is the rational Narayana number $\operatorname{Nar}(a, b ; k)$.

2. If $\pi \in \mathrm{NC}(a, b)$ and $\pi^{\prime}$ is a noncrossing partition of $[b-1]$ which coarsens $\pi$, then $\pi^{\prime} \in \mathrm{NC}(a, b)$.

When $(a, b)=(n, n+1)$, the set $\mathrm{NC}(n, n+1)$ of rational noncrossing partitions is just the set $\mathrm{NC}(n)$ of all noncrossing partitions of $[n]$. When $(a, b)=(n, m n+1)$ we have that $\mathrm{NC}(n, m n+1)$ of rational noncrossing partitions is the set of all noncrossing partitions of $[m n]$ which are $m$-divisible in the sense that every block size is divisible by $m$. Armstrong, Rhoades, and Williams posed the problem of finding an analogous 'intrinsic' characterization of $\mathrm{NC}(a, b)$ for arbitrary $a<b$ coprime. We give two such characterizations in Section 3. 


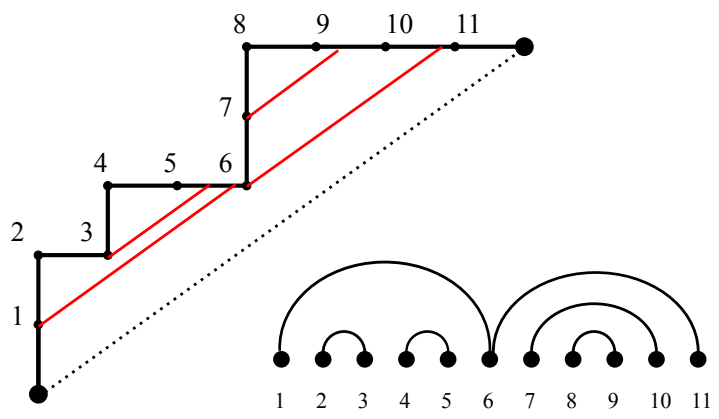

Figure 2: A 5,7-Dyck path and the corresponding 5, 7-homogeneous noncrossing partition.

\subsection{Homogeneous rational noncrossing partitions}

Rational Dyck paths are used in [4] to construct a generalization of noncrossing perfect matchings on the set $[2 n]$. The construction is similar to that of the rational noncrossing partitions.

Let $(a, b)$ be coprime and let $D$ be an $a, b$-Dyck path. We construct a set partition $\bar{\pi}(D)$ of the set $[a+b-1]$ as follows. Label the interior lattice points of $D$ from southwest to northeast with $1,2, \ldots, a+b-1$. Fire lasers of slope $\frac{a}{b}$ from every lattice point of $D$ which is at the south end of a north step (not just those lattice points which are valleys of $D$ ). The set partition $\bar{\pi}(D)$ is given by declaring $i \sim j$ if and only if the labels $i$ and $j$ are not separated by laser fire. As before, we consider labels to be slightly below their lattice points. Topological considerations make it clear that $\bar{\pi}(D)$ is a noncrossing partition of $[a+b-1]$. An example of this construction is shown in Figure 2 for $(a, b)=(5,8)$.

Considering $\bar{\pi}(D)$ for all possible $a, b$-Dyck paths $D$ gives rise to the set of $a, b$ homogeneous rational noncrossing partitions

$$
\operatorname{HNC}(a, b):=\{\bar{\pi}(D): D \text { an } a, b \text {-Dyck path }\}
$$

The adjective 'homogeneous' refers to the fact that every set partition in $\operatorname{HNC}(a, b)$ has $a$ blocks. By construction, we have that $\operatorname{HNC}(a, b) \subseteq \mathrm{NC}(a+b-1)$.

When $(a, b)=(n, n+1)$, the set $\operatorname{HNC}(n, n+1)$ is the set of noncrossing perfect matchings on $[2 n]$. When $(a, b)=(n, m n+1)$, the set $\operatorname{HNC}(n, m n+1)$ is the set of noncrossing set partitions on $[m n]$ in which every block has size $m$ (these are also called $m$-equal noncrossing partitions). Some basic facts about $\operatorname{HNC}(a, b)$ for general $a<b$ are as follows.

Proposition 2.2. Let $a<b$ be coprime positive integers.

1. The map

$$
\bar{\pi}:\{a, b-D y c k \text { paths }\} \rightarrow \operatorname{HNC}(a, b)
$$

is bijective, so that $|\operatorname{HNC}(a, b)|=\operatorname{Cat}(a, b)$.

2. The set $\operatorname{HNC}(a, b)$ is closed under the rotation operator rot. 

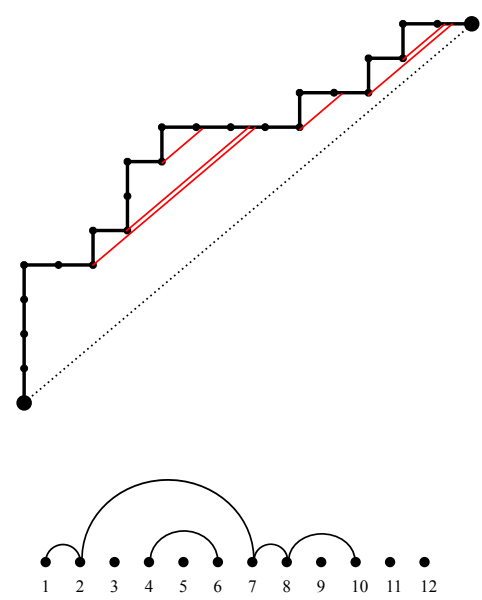
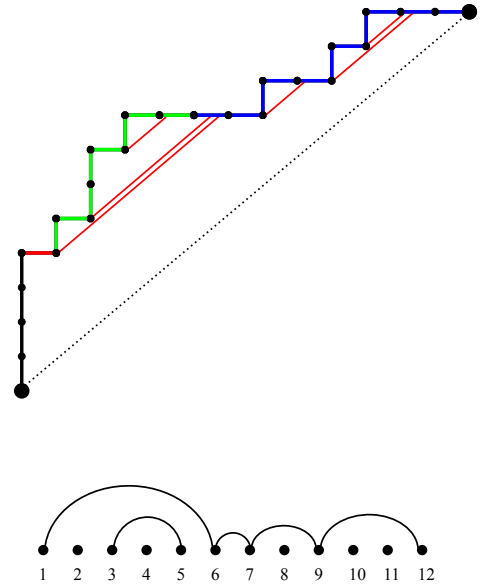
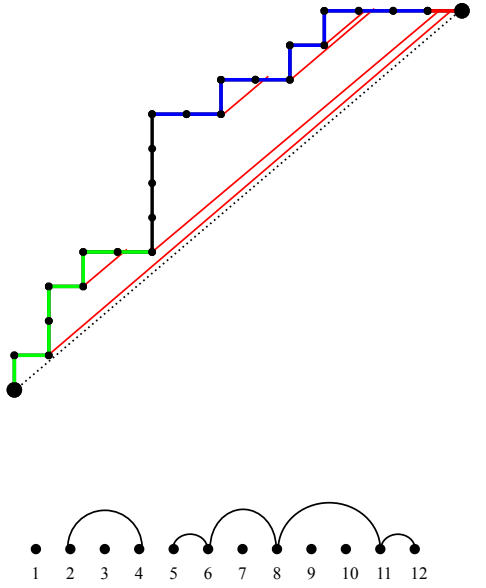

Figure 3: The action of rot' on $a, b$-Dyck paths.

In [4] a promotion operator on $a, b$-Dyck paths is shown to intertwine the action of rot with the map $\bar{\pi}$ from paths to homogeneous noncrossing partitions. We will give an inhomogeneous analog of this promotion operator in the next section.

\section{Characterizations of Rational Noncrossing Partitions}

\subsection{The action of rotation}

Let $a<b$ be coprime. In this subsection we will prove that $\mathrm{NC}(a, b)$ is closed under the rotation operator rot by describing rotation (or, rather, its inverse) as an operator on $a, b$-Dyck paths. We will define an operator

$$
\text { rot }^{\prime}:\{a, b \text {-Dyck paths }\} \longrightarrow\{a, b \text {-Dyck paths }\}
$$

on the set of $a, b$-Dyck paths which satisfies $\operatorname{rot}^{-1} \circ \pi=\pi \circ \operatorname{rot}^{\prime}$.

The definition of rot' is probably best understood visually. Figure 3 shows three 11,13-Dyck paths. The middle path is the image of the left path under rot' and the right path is the image of the middle path under rot'. The valley lasers are fired on each of these Dyck paths, and the corresponding partitions in $\mathrm{NC}(11,13)$ are shown below, but the vertex labels on the Dyck paths are omitted for legibility.

Let $D_{1}$ be the 11,13-Dyck path on the left of Figure 3. The westernmost horizontal run of $D_{1}$ has size $>1$. Because of this, we define $D_{2}:=\operatorname{rot}^{\prime}\left(D_{1}\right)$ by removing one step from this horizontal run and adding it to the easternmost horizontal run of $D_{1}$. The lattice path $D_{2}$ is displayed in the middle of Figure 3. Informally, the path $D_{2}$ is obtained from $D_{1}$ by translating one unit west.

Let $D_{2}$ be the 11,13-Dyck path in the middle of Figure 3. The westernmost horizontal run of $D_{2}$ has size 1 . The definition of $D_{3}:=\operatorname{rot}^{\prime}\left(D_{2}\right)$ is more complicated in this case. We break the lattice path $D_{2}$ up into four subpaths. The first subpath, shown in black, 
is the initial northern run of $D_{2}$. The second subpath, shown in red, is the single east step which occurs after this run. The third subpath, shown in green, extends from the westernmost valley of $D_{2}$ to the (necessarily east) step just before the laser fired from this valley hits $D_{2}$. The fourth subpath, shown in blue, extends from the end of the third subpath to the terminal point $(b, a)$ of $D_{2}$. The path $D_{3}$ is obtained by concatenating the third, first, fourth, and second subpaths, in that order. The concatenation of green, then black, then blue, then red is shown on the right of Figure 3.

More formally, given an $a, b$-Dyck path $D$, the definition of $\operatorname{rot}^{\prime}(D)$ breaks up into three cases. Let $D=N^{i_{1}} E^{j_{1}} \cdots N^{i_{m}} E^{j_{m}}$ be the decomposition of $D$ into nonempty vertical and horizontal runs.

1. If $m=1$ so that $D=N^{a} E^{b}$, we set

$$
\operatorname{rot}^{\prime}(D):=N^{a} E^{b}=D
$$

2. If $m, j_{1}>1$, we set

$$
\operatorname{rot}^{\prime}(D):=N^{i_{1}} E^{j_{1}-1} N^{i_{2}} E^{j_{2}} \cdots N^{i_{m}} E^{j_{m}+1} .
$$

3. If $m>1$ and $j_{1}=1$, let $P=\left(1, i_{1}\right)$ be the westernmost valley of $D$. The laser $\ell(P)$ fired from $P$ hits $D$ on a horizontal run $E^{i_{k}}$ for some $2 \leqslant k \leqslant m$. Suppose that $\ell(P)$ hits the horizontal run $E^{i_{k}}$ on step $r$, where $2 \leqslant r \leqslant i_{k}$. We set

$$
\operatorname{rot}^{\prime}(D):=N^{i_{2}} E^{i_{2}} \cdots N^{i_{k-1}} E^{i_{k-1}} N^{i_{k}} E^{r-1} N^{i_{1}} E^{i_{k}-r+1} N^{i_{k+1}} E^{i_{k+1}} \cdots N^{i_{m}} E^{j_{m}+1} .
$$

Proposition 3.1. The definition of rot' given above gives a well defined operator on the set of $a, b$-Dyck paths. As operators $\{a, b$-Dyck paths $\} \longrightarrow \mathrm{NC}(a, b)$, we have that $\operatorname{rot}^{-1} \circ \pi=\pi \circ \operatorname{rot}^{\prime}$. In particular, the set $\mathrm{NC}(a, b)$ is closed under rotation.

Proof. Let $D$ be an $a, b$-Dyck path. If the westernmost horizontal run of $D$ has size $>1$, it is clear that $\operatorname{rot}^{\prime}(D)$ is also an $a, b$-Dyck path and that the corresponding set partitions are related by $\operatorname{rot}^{-1}(\pi(D))=\pi\left(\operatorname{rot}^{\prime}(D)\right)$. We therefore assume that the westernmost horizontal run of $D$ consists of a single step.

We claim that the lattice path $\operatorname{rot}^{\prime}(D)$ stays above the diagonal $y=\frac{a}{b} x$. Indeed, consider the decomposition of $\operatorname{rot}^{\prime}(D)$ as in Figure 3. The first (green) subpath of $\operatorname{rot}^{\prime}(D)$ stays above $y=\frac{a}{b} x$ because the laser fired from the westernmost valley of $D$ has slope $\frac{a}{b}$. The second (black) subpath of $\operatorname{rot}^{\prime}(D)$ is a vertical run, so the concatenation of the first and second subpaths of $\operatorname{rot}^{\prime}(D)$ stay above $y=\frac{a}{b} x$. The third (blue) subpath of $\operatorname{rot}^{\prime}(D)$ is just the corresponding subpath of $D$ translated one unit west, and certainly stays above the line $y=\frac{a}{b} x$. Since the fourth (red) subpath of $\operatorname{rot}^{\prime}(D)$ is a single east step, we conclude that the entire path $\operatorname{rot}^{\prime}(D)$ stays above $y=\frac{a}{b} x$, and so is an $a, b$-Dyck path.

By the last paragraph, the set partition $\pi\left(\operatorname{rot}^{\prime}(D)\right) \in \mathrm{NC}(a, b)$ is well defined. We argue that $\operatorname{rot}^{-1}(\pi(D))=\pi\left(\operatorname{rot}^{\prime}(D)\right)$. To do this, we consider how the valley lasers of $\operatorname{rot}^{\prime}(D)$ relate to the corresponding valley lasers of $D$. 

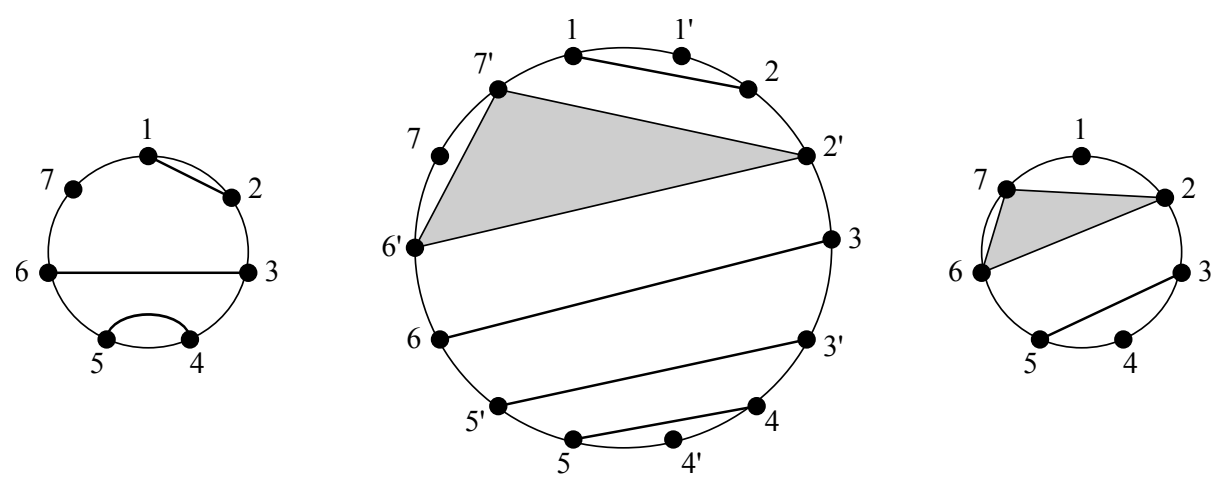

Figure 4: An example of Kreweras complement.

- The valley lasers in the third (blue) subpath of $\operatorname{rot}^{\prime}(D)$ are just the valley lasers in the corresponding subpath of $D$ shifted one unit west.

- The valley lasers in the first (green) subpath of $\operatorname{rot}^{\prime}(D)$ are either the valley lasers in the corresponding subpath of $D$ shifted one unit west, or hit $\operatorname{rot}^{\prime}(D)$ on its terminal east step, depending on whether these lasers hit $D$ in its third (green) or fourth (blue) subpaths.

- The valley laser of the westernmost valley in $D$ is replaced by the laser in the valley of $\operatorname{rot}^{\prime}(D)$ between its first (green) and second (black) subpaths, which necessarily hits $\operatorname{rot}^{\prime}(D)$ on its terminal east step.

From this description of the valley lasers of $\operatorname{rot}^{\prime}(D)$, one checks that $\operatorname{rot}^{-1}(\pi(D))=$ $\pi\left(\operatorname{rot}^{\prime}(D)\right)$, as desired.

\subsection{Characterization from Kreweras complement}

Our first characterization of rational noncrossing partitions gives a description of their Kreweras complements. Let $\pi$ be a noncrossing partition of $[n]$. The Kreweras complement $\operatorname{krew}(\pi)$ is the noncrossing partition obtained by drawing the $2 n$ vertices

$$
1,1^{\prime}, 2,2^{\prime}, \ldots, n, n^{\prime}
$$

clockwise on the boundary of a disk in that order, drawing the blocks of $\pi$ on the unprimed vertices, and letting $\mathrm{krew}(\pi)$ be the unique coarsest partition of the primed vertices which

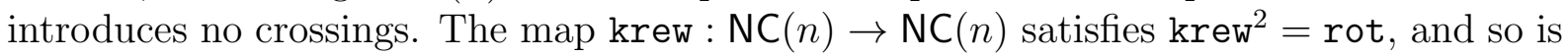
a bijection.

An example of Kreweras complementation for $n=7$ is shown in Figure 4. We have krew : $\{\{1,2\},\{3,6\},\{4,5\},\{7\}\} \mapsto\{\{1\},\{2,6,7\},\{3,5\},\{4\}\}$.

For $(a, b) \neq(n, n+1)$, the set $\mathrm{NC}(a, b)$ is not closed under Kreweras complement. Indeed, the one block set partition $\{[b-1]\}$ is contained in $\mathrm{NC}(a, b)$ but its Kreweras complement (the all singletons set partition) is not. On the other hand, the set $\mathrm{NC}(a, b)$ 
is in bijective correspondence with its Kreweras image $\mathrm{krew}(\mathrm{NC}(a, b))$. The problem of characterizing $\mathrm{NC}(a, b)$ is therefore equivalent to the problem of characterizing its Kreweras image.

Given $\pi \in \mathrm{NC}(a, b)$, there is a simple relationship between the blocks of $\operatorname{krew}(\pi) \in$ $\mathrm{NC}(b-1)$ and the laser set $\ell(D)$ of the $a, b$-Dyck path $D$ corresponding to $\pi$.

Lemma 3.2. Let $a<b$ be coprime, let $\pi \in \mathrm{NC}(a, b)$ have corresponding $a, b$-Dyck path $D$, and let $\mathrm{krew}(\pi)$ be the Kreweras complement of $\pi$. The laser set $\ell(D)$ is

$$
\ell(D)=\{(i, \max (B)): B \text { is a block of } \mathrm{krew}(\pi) \text { and } i \text { is a nonmaximal element of } B\} \text {. }
$$

Proof. This is clear from the topological definition of $\pi(D)$ and Kreweras complement.

Let $\pi \in \mathrm{NC}(b-1)$ be a noncrossing partition and suppose we would like to determine whether $\pi$ is in $\mathrm{NC}(a, b)$. In light of Lemma 3.2, it is enough to know whether there exists an $a, b$-Dyck path $D$ whose laser set consists of pairs $(i, \max (B))$, where $i \in B$ runs over all nonmaximal elements of all blocks $B$ of $\operatorname{krew}(\pi)$. A characterization of the laser sets of $a, b$-Dyck paths can be read off from the results of [11]. In [11] this characterization was used to prove that the rational analog of the associahedron is a flag simplicial complex.

Proposition 3.3. Let $a<b$ be coprime and let $\pi$ be a noncrossing partition of $[b-1]$. Then $\pi$ is an a,b-noncrossing partition if and only if for every block $B$ of the Kreweras complement $\mathrm{krew}(\pi)$ we have

- $(i, \max (B)) \in A(a, b)$ for every nonmaximal element $i \in B$, and

- for any two nonmaximal elements $i<j$ in $B$,

$$
\left\lceil(\max (B)-i) \frac{a}{b}\right\rceil-\left\lceil(\max (B)-j) \frac{a}{b}\right\rceil>(j-i) \frac{a}{b} .
$$

Proof. As mentioned above, we know that $\pi \in \mathrm{NC}(a, b)$ if and only if there exists an $a, b$ Dyck path $D$ whose laser set consists of all possible pairs $(i, \max (B))$, where $B$ ranges over all blocks of krew $(\pi)$ and $i$ ranges over all nonmaximal elements of $B$. For such an $a, b$-Dyck path $D$ to exist, it is certainly necessary that $(i, \max (B)) \in A(a, b)$ always, so we assume this condition holds.

By [11, Proposition 1.3] and [11, Lemma 4.3], we know that an $a, b$-Dyck path $D$ as in the previous paragraph exists if and only if for every two pairs $(i, \max (B))$ and $\left(i^{\prime}, \max \left(B^{\prime}\right)\right)$, there exists an $a, b$-Dyck path $\bar{D}$ with laser set

$$
\ell(\bar{D})=\left\{(i, \max (B)),\left(j, \max \left(B^{\prime}\right)\right)\right\} .
$$

By [11, Lemma 5.3] and [11, Proposition 5.5], such an $a, b$-Dyck path $\bar{D}$ does not exist if and only if $\max (B)=\max \left(B^{\prime}\right)$ (so that $B=B^{\prime}$ ) and

$$
\left\lceil(\max (B)-i) \frac{a}{b}\right\rceil-\left\lceil(\max (B)-j) \frac{a}{b}\right\rceil<(j-i) \frac{a}{b},
$$

where $i<j$. Since $1 \leqslant i<j<b-1$, we have $\left\lceil(\max (B)-i) \frac{a}{b}\right\rceil-\left\lceil(\max (B)-j) \frac{a}{b}\right\rceil \neq$ $(j-i) \frac{a}{b}$ and the result follows. 
Example 3.4. Let $(a, b)=(5,8)$ and consider the following three partitions in $\mathrm{NC}(7)$ :

$$
\begin{aligned}
& \pi_{1}:=\{\{1\},\{2,6,7\},\{3\},\{4,5\}\}, \\
& \pi_{2}:=\{\{1\},\{2,6,7\},\{3,4\},\{5\}\}, \\
& \pi_{3}:=\{\{1\},\{2,7\},\{3,4,5\},\{6\}\} .
\end{aligned}
$$

To determine which of $\pi_{1}, \pi_{2}, \pi_{3}$ belong to $\mathrm{NC}(5,8)$, we start by computing their Kreweras images:

$$
\begin{aligned}
& \operatorname{krew}\left(\pi_{1}\right):=\{\{1,7\},\{2,3,5\},\{4\},\{6\}\}, \\
& \operatorname{krew}\left(\pi_{2}\right):=\{\{1,7\},\{2,4,5\},\{3\},\{6\}\}, \\
& \operatorname{krew}\left(\pi_{3}\right):=\{\{1,7\},\{2,5,6\},\{3\},\{4\}\} .
\end{aligned}
$$

Since $\{2,3,5\}$ is a block of $\mathrm{krew}\left(\pi_{1}\right)$ and $(3,5) \notin A(5,8)$, we conclude that $\pi_{1} \notin \mathrm{NC}(5,8)$. Since $\{2,4,5\}$ is a block of $\mathrm{krew}\left(\pi_{2}\right)$ and $\left\lceil(5-2) \frac{5}{8}\right\rceil-\left\lceil(5-4) \frac{5}{8}\right\rceil \leqslant(4-2) \frac{5}{8}$, we conclude that $\pi_{2} \notin \mathrm{NC}(5,8)$. Since both of the bullets in Proposition 3.3 hold for $\pi_{3}$, we conclude that $\pi_{3} \in \mathrm{NC}(5,8)$.

\section{$3.3 \quad a, b-r a n k s$}

In order to state our second characterization of $a, b$-noncrossing partitions, we introduce a new way to measure the 'size' of a block $B$ of a noncrossing partition $\pi$ other than its cardinality $|B|$. Our rational analog of block size is as follows.

Definition 3.5. Let $a<b$ be coprime positive integers and let $\pi \in \mathrm{NC}(b-1)$ be $a$ noncrossing partition of $[b-1]$. We assign an integer $\operatorname{rank}_{a, b}^{\pi}(B) \in \mathbb{Z}$ to every block $B$ of $\pi$ (or just $\operatorname{rank}(B)$ when $a, b$, and $\pi$ are clear from context) by the following recursive procedure. Let $\preceq$ be the partial order on the blocks of $\pi$ defined by

$$
B^{\prime} \preceq B \Longleftrightarrow\left[\min \left(B^{\prime}\right), \max \left(B^{\prime}\right)\right] \subseteq[\min (B), \max (B)] .
$$

The integers $\operatorname{rank}_{a, b}^{\pi}(B)$ are implicitly determined by the formula

$$
\sum_{B^{\prime} \preceq B} \operatorname{rank}_{a, b}^{\pi}\left(B^{\prime}\right)=\left\lceil(\max (B)-\min (B)+1) \frac{a}{b}\right\rceil,
$$

for all blocks $B \in \pi$.

While we define $\operatorname{rank}_{a, b}^{\pi}(\cdot)$ as a function on the blocks of an arbitrary noncrossing partition of $[b-1]$, this notion of rank will be more useful for $a, b$-noncrossing partitions. We will see that rank is more useful than cardinality as a block size measure in rational Catalan theory. This subsection proves basic properties of the function $\operatorname{rank}_{a, b}^{\pi}(\cdot)$ on the blocks of $a, b$-noncrossing partitions.

In the Fuss-Catalan case $b \equiv 1(\bmod a)$, rank and cardinality determine each other. 
Proposition 3.6. Let $(a, b)=(n, m n+1)$, let $\pi \in \mathrm{NC}(n, m n+1)$ be an $m$-divisible noncrossing partition of $[\mathrm{mn}]$, and let $B$ be a block of $\pi$. We have

$$
\operatorname{rank}_{n, m n+1}^{\pi}(B)=\frac{|B|}{m} \text {. }
$$

Proof. For any block $B$ of $\pi$, we have $1 \leqslant \min (B) \leqslant \max (B) \leqslant m n$ and the divisibility relation $m \mid(\max (B)-\min (B)+1)$, so that

$$
\left\lceil(\max (B)-\min (B)+1) \frac{n}{m n+1}\right\rceil=\frac{\max (B)-\min (B)+1}{m} .
$$

The assignment $B \mapsto \frac{|B|}{m}$ therefore satisfies the recursion for $\operatorname{rank}_{n, m n+1}^{\pi}(\cdot)$.

For general $a<b$ coprime, the $a, b$-rank of a block of an $a, b$-noncrossing partition $\pi \in \mathrm{NC}(a, b)$ is not necessarily determined by its cardinality. For example, consider $(a, b)=(3,5)$. Then $\pi=\{\{1,3\},\{2\},\{4\}\} \in \mathrm{NC}(3,5)$. We have

$$
\operatorname{rank}_{3,5}^{\pi}(\{1,3\})=\operatorname{rank}_{3,5}^{\pi}(\{2\})=\operatorname{rank}_{3,5}^{\pi}(\{4\})=1 .
$$

Proposition 3.6 shows that the divergence between rank and cardinality is a genuinely new feature of rational Catalan combinatorics and is invisible at the Catalan and Fuss-Catalan levels of generality.

Definition 3.7. If $\pi \in \mathrm{NC}(a, b)$ is an $a, b$-noncrossing partition, the rank sequence $R(\pi)=$ $\left(r_{1}, \ldots, r_{b-1}\right)$ of $\pi$ is the sequence of nonnegative integers given by

$$
r_{i}:= \begin{cases}\operatorname{rank}(B) & \text { if } i=\min (B) \text { for some block } B \text { of } \pi \\ 0 & \text { otherwise. }\end{cases}
$$

For example, the rank sequence of the 5,8-noncrossing partition shown in Figure 1 has rank sequence $(1,1,0,2,1,0,0)$. Observe that this is also the sequence of vertical runs of the corresponding 5,8-Dyck path, so that the rank sequence is equivalent to the Dyck path. This is a general phenomenon.

Proposition 3.8. Let $a<b$ be coprime, let $\pi$ be an $a, b$-noncrossing partition with rank sequence $R(\pi)=\left(r_{1}, \ldots, r_{b-1}\right)$, and let $D$ be the $a, b$-Dyck path associated to $\pi$.

1. For any block $B$ of $\pi$, the vertical run of $D$ visible from $\pi$ has size $\operatorname{rank}(B)$.

2. The Dyck path $D$ is given by

$$
D=N^{r_{1}} E N^{r_{2}} E \cdots N^{r_{b-1}} E E .
$$


Proof. For any block $B$ of $\pi$, we have that $\min (B)$ labels the lattice point just to the right of the vertical run labeled by $B$. Therefore, (2) follows from (1).

To prove (1), recall the partial order $\preceq$ on the blocks of $\pi$. If $B$ is minimal with respect to $\preceq$, then $B=[\min (B), \max (B)]$ is an interval. The labels of $B$ must appear on a single horizontal run of $D$, say on the line $y=c$. Let $i_{B}$ be the length of the vertical run visible from $B$, let $P$ be the valley at the bottom of this vertical run, and let $\ell(P)$ be the laser fired from $P$.

The laser $\ell(P)$ must intersect the line $y=c$. We claim that it does so in the open $x$-interval $(\max (B), \max (B)+1)$. Indeed, by coprimality there exists a unique lattice point $Q$ on $D$ which is northwest of the laser $\ell(P)$ and has minimum horizontal distance to the laser $\ell(P)$. Let $m$ be the $x$-coordinate of $Q$. We claim that $m \in B$. Indeed, if $m \notin B$, there must be a laser $\ell\left(Q^{\prime}\right)$ fired from a valley $Q^{\prime}$ which separates $m$ from $B$. But then $Q^{\prime}$ would be closer than $Q$ to $\ell(P)$, so we conclude that $m \in B$. Since $B=[\min (B), \max (B)]$ is an interval, we have that $\ell(P)$ intersects $y=c$ in the open $x$-interval $(\max (B), \max (B)+1)$. This implies that $\operatorname{rank}(B)=i_{B}$, as desired.

Now suppose that $B$ is not $\preceq$-minimal among the blocks of $\pi$. Then the interval $[\min (B), \max (B)]$ is a union of at least two blocks of $\pi$. For any block $B^{\prime}$ contained in this interval, let $i_{B^{\prime}}$ denote the size of the vertical run visible from $B^{\prime}$. We may inductively assume that, for all $B^{\prime} \neq B$, we have

$$
\operatorname{rank}_{a, b}^{\pi}\left(B^{\prime}\right)=i_{B^{\prime}}
$$

Let $\pi_{0}$ be the set partition obtained from $\pi$ by merging the blocks contained in $[\min (B), \max (B)]$. Then $\pi_{0}$ is noncrossing, and hence $a, b$-noncrossing by Proposition 2.1 (2). The recursion for rank says that

$$
\operatorname{rank}_{a, b}^{\pi_{0}}([\min (B), \max (B)])=\sum_{B^{\prime} \subseteq[\min (B), \max (B)]} \operatorname{rank}_{a, b}^{\pi}(B)=\operatorname{rank}_{a, b}^{\pi}(B)+\sum_{\substack{B^{\prime} \subseteq[\min (B), \max (B)] \\ B^{\prime} \neq B}} i_{B^{\prime}},
$$

where the second equality used the inductive hypothesis. Moreover, the Dyck path $D_{0}$ corresponding to $\pi_{0}$ is obtained from $D$ by replacing the portion between the $x$ coordinates $\min (B)$ and $\max (B)$ with a single vertical run, followed by a single horizontal run. In particular, the size of the vertical run in $D_{0}$ visible from $[\min (B), \max (B)]$ is $\sum_{B^{\prime} \subseteq[\min (B), \max (B)]} i_{B^{\prime}}$. This forces $\operatorname{rank}_{a, b}^{\pi}(B)=i_{B}$, as desired.

The cardinality function $|\cdot|$ on blocks of noncrossing partitions satisfies the following two properties.

- Let $\pi$ and $\pi^{\prime}$ be noncrossing partitions such that $\pi$ refines $\pi^{\prime}$. For any block $B^{\prime}$ of $\pi^{\prime}$, write $B^{\prime}=B_{1} \uplus \cdots \uplus B_{k}$, where $B_{1}, \ldots, B_{k}$ are blocks of $\pi$. We have

$$
\left|B^{\prime}\right|=\left|B_{1}\right|+\cdots+\left|B_{k}\right| \text {. }
$$

- The function $|\cdot|$ on blocks of noncrossing set partitions is invariant under the dihedral action of $\langle\operatorname{rot}, \mathrm{rfn}\rangle$. 
To justify our intuition that rank measures size, we prove that $\operatorname{rank}_{a, b}^{\pi}(\cdot)$ enjoys the same properties on the set of $a, b$-noncrossing partitions.

Proposition 3.9. Let $a<b$ be coprime, let $\pi, \pi^{\prime} \in \mathrm{NC}(a, b)$, and assume that $\pi$ refines $\pi^{\prime}$. Let $B^{\prime}$ be a block of $\pi^{\prime}$ and let $B_{1}, \ldots, B_{k}$ be the blocks of $\pi$ such that $B^{\prime}=B_{1} \uplus \cdots \uplus B_{k}$. We have

$$
\operatorname{rank}_{a, b}^{\pi^{\prime}}\left(B^{\prime}\right)=\operatorname{rank}_{a, b}^{\pi}\left(B_{1}\right)+\cdots+\operatorname{rank}_{a, b}^{\pi}\left(B_{k}\right) .
$$

Proof. It suffices to consider the case where $\pi^{\prime}$ is obtained by merging two blocks of $\pi$, say $B_{1}$ and $B_{2}$. Suppose $\min \left(B_{1}\right)<\min \left(B_{2}\right)$. If $D, D^{\prime}$ are the $a, b$-Dyck paths corresponding to $\pi, \pi^{\prime}$, then $D^{\prime}$ is obtained from $D$ by moving the vertical run visible from $B_{2}$ on top of the vertical run visible from $B_{1}$. The merged vertical run in $D^{\prime}$ is visible from $B_{1} \uplus B_{2}$. The result follows from Proposition 3.8.

Proposition 3.9 fails for partitions $\pi, \pi \in \mathrm{NC}(b-1)$ which are not both $a$, $b$-noncrossing. For example, let $(a, b)=(2,5), \pi=\{\{1,2,3\},\{4\}\}$, and $\pi^{\prime}=\{\{1,2,3,4\}\}$. We have $\operatorname{rank}_{2,5}^{\pi}(\{1,2,3\})=2 \operatorname{rank}_{2,5}^{\pi}(\{4\})=1$, and $\operatorname{rank}_{2,5}^{\pi^{\prime}}(\{1,2,3,4\})=2$.

Proposition 3.10. Let $a<b$ be coprime, let $\pi \in \mathrm{NC}(a, b)$, and let $B$ be a block of $\pi$. We have

$$
\begin{aligned}
\operatorname{rank}_{a, b}^{\pi}(B) & =\operatorname{rank}_{a, b}^{\operatorname{rot}(\pi)}(\operatorname{rot}(B)), \\
\operatorname{rank}_{a, b}^{\pi}(B) & =\operatorname{rank}_{a, b}^{\operatorname{rnn}(\pi)}(\operatorname{rfn}(B)) .
\end{aligned}
$$

Proof. Since the intervals $[\min (B), \max (B)]$ and $[\min (\operatorname{rfn}(B)), \max (\operatorname{rfn}(B))]$ have the same length, reflection invariance is clear (and still holds if $\pi$ fails to be $a, b$-noncrossing).

Recall that rot acts by adding 1 to every index, modulo $b-1$. Rotation invariance is therefore clear unless $B$ has the form $B=\left\{i_{1}<\cdots<i_{j}<b-1\right\}$ with $i_{1}>1$. In this case, the intervals $\left[1, i_{1}-1\right],\left[i_{2}+1, i_{3}-1\right], \ldots,\left[i_{j}+1, b-2\right]$ must each be unions of blocks of $\pi$. Let $B_{1}, \ldots, B_{r}$ be a complete list of these remaining blocks. We certainly have $\operatorname{rank}_{a, b}^{\pi}\left(B_{i}\right)=\operatorname{rank}_{a, b}^{\operatorname{rot}(\pi)}\left(\operatorname{rot}\left(B_{i}\right)\right)$ for $1 \leqslant i \leqslant r$. On the other hand, Proposition 3.1 and the assumption that $\pi \in \mathrm{NC}(a, b)$ guarantee that $\operatorname{rot}(\pi) \in \mathrm{NC}(a, b)$. Proposition 3.8 shows that

$$
\begin{aligned}
a & =\operatorname{rank}_{a, b}^{\pi}(B)+\operatorname{rank}_{a, b}^{\pi}\left(B_{1}\right)+\cdots+\operatorname{rank}_{a, b}^{\pi}\left(B_{r}\right) \\
& =\operatorname{rank}_{a, b}^{\operatorname{rot}(\pi)}(\operatorname{rot}(B))+\operatorname{rank}_{a, b}^{\operatorname{rot}(\pi)}\left(\operatorname{rot}\left(B_{1}\right)\right)+\cdots+\operatorname{rank}_{a, b}^{\operatorname{rot}(\pi)}\left(\operatorname{rot}\left(B_{r}\right)\right) .
\end{aligned}
$$

Since $\operatorname{rank}_{a, b}^{\pi}\left(B_{i}\right)=\operatorname{rank}_{a, b}^{\operatorname{rot}(\pi)}\left(\operatorname{rot}\left(B_{i}\right)\right)$ for $1 \leqslant i \leqslant r$, we have that $\operatorname{rank}_{a, b}^{\pi}(B)=$ $\operatorname{rank}_{a, b}^{\operatorname{rot}(\pi)}(\operatorname{rot}(B))$.

Proposition 3.10 does not hold for partitions $\pi \in \mathrm{NC}(b-1)$ which are not $a, b$ noncrossing. For example, take $(a, b)=(2,5)$ and $\pi=\{\{1\},\{2,3,4\}\}$, so that $\operatorname{rot}(\pi)=$ $\{\{1,3,4\}\{2\}\}$. We have $\operatorname{rank}_{2,5}^{\pi}(\{2,3,4\})=2 \operatorname{but}_{\operatorname{rank}_{2,5}^{\text {rot }}(\pi)}(\{1,3,4\})=1$. 


\subsection{Characterization from rank function}

The $a, b$-rank function $\operatorname{rank}_{a, b}^{\pi}(\cdot)$ can be used to decide whether a given noncrossing partition $\pi \in \mathrm{NC}(b-1)$ is $a, b$-noncrossing. This is our first application of $a, b$-rank to rational Catalan theory. To begin, we introduce the notion of a valid $a, b$-ranking.

Definition 3.11. Let $\pi \in \mathrm{NC}(b-1)$ be an arbitrary noncrossing partition of $[b-1]$. We say that $\pi$ has a valid $a, b$-ranking if $\operatorname{rank}_{a, b}^{\pi}(B)>0$ for every block $B \in \pi$ and

$$
\sum_{B \in \pi} \operatorname{rank}(B)=a
$$

Example 3.12. Let $(a, b)=(3,5)$ and consider the two noncrossing partitions

$$
\pi_{1}=\{\{1,2,4\},\{3\}\}, \pi_{2}=\{\{1,2\},\{3\},\{4\}\} \in \mathrm{NC}(4) .
$$

Then $\pi_{1}$ has a valid 3,5-ranking, but $\pi_{2}$ does not. Indeed, we have

$$
\begin{aligned}
& \operatorname{rank}_{3,5}^{\pi_{2}}(\{1,2\})+\operatorname{rank}_{3,5}^{\pi_{2}}(\{3\})+\operatorname{rank}_{3,5}^{\pi_{2}}(\{4\})=2+1+1>3 . \\
& \text { If }(a, b)=(4,7) \text { and } \\
& \qquad \pi_{3}=\{\{1,6\},\{2\},\{3\},\{4\},\{5\}\} \in \mathrm{NC}(6),
\end{aligned}
$$

then we have $\operatorname{rank}_{4,7}^{\pi_{3}}(\{1,6\})=0$, so that $\pi_{3}$ does not have a valid 4,7-ranking.

We record the following lemma for future use.

Lemma 3.13. Let $a<b$ be coprime and let $\pi \in N C(b-1)$ be an arbitrary noncrossing partition of $[b-1]$. We have

$$
\sum_{B \in \pi} \operatorname{rank}(B) \geqslant a
$$

Proof. Recall the partial order $\preceq$ on the blocks of $\pi$. If $B^{\prime}$ is maximal with respect to $\preceq$, we have

$$
\sum_{B \preceq B^{\prime}} \operatorname{rank}(B)=\left\lceil\left(\max \left(B^{\prime}\right)-\min \left(B^{\prime}\right)+1\right) \frac{a}{b}\right\rceil>\left(\max \left(B^{\prime}\right)-\min \left(B^{\prime}\right)+1\right) \frac{a}{b} .
$$

Summing over all $\preceq$-maximal blocks $B^{\prime}$ gives

$$
\sum_{B \in \pi} \operatorname{rank}(B)>(b-1) \frac{a}{b}
$$

which is equivalent to the desired inequality since $\sum_{B \in \pi} \operatorname{rank}(B) \in \mathbb{Z}$. 
By Proposition 3.8, every $a, b$-noncrossing partition has a valid $a, b$-ranking. It would be nice if the converse held, but it does not; if $(a, b)=(2,5)$, the partition

$$
\pi=\{\{1,2,4\},\{3\}\}
$$

has a valid 2,5 -ranking but $\pi \notin \mathrm{NC}(2,5)$. Our second characterization of $a, b$-noncrossing partitions states that $\pi \in \mathrm{NC}(a, b)$ if and only if every element in the orbit of $\pi$ under rotation has a valid $a, b$-ranking. To prove this statement, we start by examining how validity behaves under coarsening.

In general, having a valid $a, b$-ranking is not closed under coarsening of noncrossing partitions. For example, let $(a, b)=(5,11)$ and consider the two partitions $\pi, \pi^{\prime} \in \mathrm{NC}(10)$ given by

$$
\begin{aligned}
\pi & =\{\{1,6,7,8,9,10\},\{2\},\{3\},\{4\},\{5\}\}, \\
\pi^{\prime} & =\{\{1,6,7,8,9,10\},\{2,5\},\{3\},\{4\}\} .
\end{aligned}
$$

Then $\pi$ has a valid 5,11-ranking but $\pi^{\prime}$ does not. On the other hand, certain types of coarsening do preserve validity.

Lemma 3.14. Let $a<b$ be coprime and let $\pi \in \mathrm{NC}(b-1)$ be a noncrossing partition such that $\pi$ has a valid $a, b$-ranking. Suppose that $\pi^{\prime} \in \mathrm{NC}(b-1)$ is another noncrossing partition obtained from $\pi$ by one of the following two operations:

1. merging two blocks $B_{0}, B_{1}$ of $\pi$ with $\min \left(B_{1}\right)=\max \left(B_{0}\right)+1$, or

2. merging two blocks $B_{0}, B_{1}$ of $\pi$ with $B_{0} \prec B_{1}$.

The noncrossing partition $\pi^{\prime}$ has a valid a,b-ranking.

Observe that if $\pi, \pi^{\prime} \in \mathrm{NC}(b-1)$ are such that $\pi$ refines $\pi^{\prime}$ and $\pi^{\prime}$ is a union of intervals, then $\pi^{\prime}$ can be obtained from $\pi$ by a sequence of coarsenings as described in Lemma 3.14.

Proof. First assume that $\pi^{\prime}$ is obtained from $\pi$ by replacing $B_{0}$ and $B_{1}$ with $B_{0} \cup B_{1}$ as in Condition 1. For $i=0,1$, let $r_{i}$ denote the sum of the ranks of the blocks $B$ of $\pi$ satisfying $B \prec B_{i}$. By the definition of rank, we have

$$
\operatorname{rank}_{a, b}^{\pi}\left(B_{i}\right)+r_{i}=\left\lceil\left(\max \left(B_{i}\right)-\min \left(B_{i}\right)+1\right) \frac{a}{b}\right\rceil
$$

for $i=0,1$. Adding these two equations together and recalling that $\lceil x\rceil+\lceil y\rceil-1 \leqslant$ $\lceil x+y\rceil \leqslant\lceil x\rceil+\lceil y\rceil$ for any $x, y \in \mathbb{R}$, we get

$$
\operatorname{rank}_{a, b}^{\pi}\left(B_{0}\right)+\operatorname{rank}_{a, b}^{\pi}\left(B_{1}\right)-1 \leqslant \operatorname{rank}_{a, b}^{\pi^{\prime}}\left(B_{0} \cup B_{1}\right) \leqslant \operatorname{rank}_{a, b}^{\pi}\left(B_{0}\right)+\operatorname{rank}_{a, b}^{\pi}\left(B_{1}\right) .
$$

Since $\pi$ has a valid $a, b$-ranking, we have $\operatorname{rank}_{a, b}^{\pi}\left(B_{i}\right)>0$ for $i=0,1$, so that $\operatorname{rank}_{a, b}^{\pi^{\prime}}\left(B_{0} \cup\right.$ $\left.B_{1}\right)>0$. Our analysis breaks up into two cases depending on whether $B_{0} \cup B_{1}$ is a $\preceq$-maximal block of $\pi^{\prime}$. 
If $B_{0} \cup B_{1}$ is a $\preceq$-maximal block of $\pi^{\prime}$, for every block $B$ of $\pi^{\prime}$ with $B \neq B_{0}, B_{1}$ we have $\operatorname{rank}_{a, b}^{\pi^{\prime}}(B)=\operatorname{rank}_{a, b}^{\pi}(B)$, so that the chain of inequalities in (9) give $\sum_{B \in \pi^{\prime}} \operatorname{rank}_{a, b}^{\pi^{\prime}}(B) \leqslant a$, so $\sum_{B \in \pi^{\prime}} \operatorname{rank}_{a, b}^{\pi^{\prime}}(B)=a$ by Lemma 3.13. Since every block of $\pi^{\prime}$ has a positive rank, we conclude that $\pi^{\prime}$ has a valid $a, b$-ranking.

If $B_{0} \cup B_{1}$ is not a $\preceq$-maximal block of $\pi^{\prime}$, there exists a unique block $B_{2} \in \pi^{\prime}$ such that $B_{2}$ covers $B_{0} \cup B_{1}$ in $\preceq$. The recursion for $\operatorname{rank}_{a, b}^{\pi^{\prime}}(\cdot)$ gives

$$
\operatorname{rank}_{a, b}^{\pi^{\prime}}\left(B_{0} \cup B_{1}\right)+r_{0}+r_{1}+r_{2}+\operatorname{rank}_{a, b}^{\pi^{\prime}}\left(B_{2}\right)=\left\lceil\left(\max \left(B_{2}\right)-\min \left(B_{2}\right)+1\right) \frac{a}{b}\right\rceil,
$$

where $r_{2}$ is the sum of the ranks of all of the blocks $B$ of $\pi^{\prime}$ satisfying $B \prec B_{2}$ but $B \npreceq B_{0}, B_{1}$. Combining (10) with the inequalities in (9), we see that $\operatorname{rank}_{a, b}^{\pi^{\prime}}\left(B_{2}\right) \geqslant$ $\operatorname{rank}_{a, b}^{\pi}\left(B_{2}\right)>0$. Since

$$
\sum_{\substack{B \in \pi \\ B \preceq B_{2}}} \operatorname{rank}_{a, b}^{\pi}(B)=\left\lceil\left(\max \left(B_{2}\right)-\min \left(B_{2}\right)+1\right) \frac{a}{b}\right\rceil=\sum_{\substack{B^{\prime} \in \pi^{\prime} \\ B^{\prime} \preceq B_{2}}} \operatorname{rank}_{a, b}^{\pi^{\prime}}\left(B^{\prime}\right),
$$

we have $\operatorname{rank}_{a, b}^{\pi^{\prime}}(B)=\operatorname{rank}_{a, b}^{\pi}(B)$ for all blocks $B \neq B_{0}, B_{1}, B_{2}$. In particular, the rank of every block of $\pi^{\prime}$ is positive and $\sum_{B \in \pi^{\prime}} \operatorname{rank}_{a, b}^{\pi^{\prime}}(B)=a$. We conclude that $\pi^{\prime}$ has a valid $a, b$-ranking.

Now assume that $\pi^{\prime}$ is obtained from $\pi$ by replacing $B_{0}$ and $B_{1}$ with $B_{0} \cup B_{1}$ as in Condition 2. It follows that $B_{1}$ covers $B_{0}$ in the $\preceq$-order. By the recursion for rank,

$$
\operatorname{rank}_{a, b}^{\pi^{\prime}}\left(B_{0} \cup B_{1}\right)=\operatorname{rank}_{a, b}^{\pi}\left(B_{0}\right)+\operatorname{rank}_{a, b}^{\pi}\left(B_{1}\right)
$$

and the ranks of all other blocks of $\pi^{\prime}$ equal the corresponding ranks in $\pi^{\prime}$, so that $\pi^{\prime}$ has a valid $a, b$-ranking.

We are ready to prove our $a, b$-rank characterization of $a, b$-noncrossing partitions.

Proposition 3.15. Let $a<b$ be coprime and let $\pi$ be a noncrossing partition of $[b-1]$. Then $\pi$ is an a,b-noncrossing partition if and only if every partition in the orbit of $\pi$ under rotation has a valid a,b-ranking.

Proof. Suppose $\pi \in \mathrm{NC}(a, b)$. By Proposition 3.1, we know that the rotation orbit of $\pi$ is contained in $\mathrm{NC}(a, b)$, so that every partition in this orbit has a valid $a, b$-ranking.

For the converse, suppose that $\pi \in \mathrm{NC}(b-1)-\mathrm{NC}(a, b)$. We argue that some partition in the rotation orbit of $\pi$ does not have a valid $(a, b)$-ranking.

Consider the Kreweras complement $\operatorname{krew}(\pi)$. If there is a block $B \in \operatorname{krew}(\pi)$ and an index $i \in B-\{\max (B)\}$ such that $(i, \max (B)) \notin A(a, b)$, then $\pi$ refines the two-block set partition $\pi^{\prime}:=\{[i+1, \max (B)],[b-1]-[i+1, \max (B)]\}$ and $\operatorname{rot}^{-i}(\pi) \operatorname{refines} \operatorname{rot}^{-i}\left(\pi^{\prime}\right)=$ $\{[1, \max (B)-i],[\max (B)-i+1, b-1]\}$. Since $\operatorname{rot}^{-i}(\pi)$ consists of two intervals, we have that $\operatorname{rot}^{-i}\left(\pi^{\prime}\right)$ can be obtained from $\operatorname{rot}^{-i}(\pi)$ by a sequence of coarsenings as in Lemma 3.14. The condition $(i, \max (B)) \notin A(a, b)$ means that

$$
\left\lceil(\max (B)-i) \frac{a}{b}\right\rceil+\left\lceil(b-\max (B)+i-1) \frac{a}{b}\right\rceil>a,
$$


so that $\operatorname{rot}^{-i}\left(\pi^{\prime}\right)$ does not have a valid $a, b$-ranking. By Lemma 3.14, we conclude that $\operatorname{rot}^{-i}(\pi)$ does not have a valid $a, b$-ranking.

By the last paragraph, we may assume that for every block $B \in \operatorname{krew}(\pi)$ and every index $i \in B-\{\max (B)\}$, we have $(i, \max (B)) \in A(a, b)$. Since $\pi \in \mathrm{NC}(b-1)-\mathrm{NC}(a, b)$, by Proposition 3.3 there exists a block $B \in \operatorname{krew}(\pi)$ and indices $i, j \in B-\{\max (B)\}$ with $i<j$ such that

$$
\left\lceil(k-i) \frac{a}{b}\right\rceil-\left\lceil(k-j) \frac{a}{b}\right\rceil<(j-i) \frac{a}{b},
$$

where $\max (B)=k$. The set partition $\pi$ refines the three-block noncrossing partition $\pi^{\prime}:=\{[i+1, j],[j+1, k],[b-1]-[i+1, k]\}$. Therefore, the set partition $\operatorname{rot}^{-i}(\pi)$ refines $\operatorname{rot}^{-i}\left(\pi^{\prime}\right)=\{[1, j-i],[j-i+1, k-i],[k-i+1, b-1]\}$. Since $\operatorname{rot}^{-i}\left(\pi^{\prime}\right)$ consists of three intervals, we have that $\operatorname{rot}^{-i}\left(\pi^{\prime}\right)$ can be obtained from $\operatorname{rot}^{-i}(\pi)$ by a sequence of coarsenings as in Lemma 3.14. We show that $\operatorname{rot}^{-i}\left(\pi^{\prime}\right)$ does not have a valid $a, b$ ranking; by Lemma 3.14, this implies that $\operatorname{rot}^{-i}(\pi)$ does not have a valid $a, b$-ranking and completes the proof.

Working towards a contradiction, suppose $\pi^{\prime \prime}:=\operatorname{rot}^{-i}\left(\pi^{\prime}\right)$ has a valid $a, b$-ranking. Denote the blocks of $\pi^{\prime \prime}$ by $B_{1}=[1, j-i], B_{2}=[j-i+1, k-i]$, and $B_{3}=[k-i+1, b-1]$. We have $\operatorname{rank}_{a, b}^{\pi^{\prime \prime}}\left(B_{1}\right)+\operatorname{rank}_{a, b}^{\pi^{\prime \prime}}\left(B_{2}\right)+\operatorname{rank}_{a, b}^{\pi^{\prime \prime}}\left(B_{3}\right)=a$. On the other hand, by Lemma 3.14, we have that $\pi^{\prime \prime \prime}:=\left\{B_{1} \cup B_{2}, B_{3}\right\}$ also has a valid $a, b$-ranking. Moreover, we have $\operatorname{rank}_{a, b}^{\pi^{\prime \prime \prime}}\left(B_{3}\right)=\operatorname{rank}_{a, b}^{\pi^{\prime \prime}}\left(B_{3}\right)$. This implies that

$$
a=\operatorname{rank}_{a, b}^{\pi^{\prime \prime \prime}}\left(B_{1} \cup B_{2}\right)+\operatorname{rank}^{\pi^{\prime \prime \prime}}\left(B_{3}\right)=\left\lceil(k-i) \frac{a}{b}\right\rceil+\operatorname{rank}^{\pi^{\prime \prime}}\left(B_{3}\right) .
$$

Putting these facts together gives

$$
\begin{aligned}
a & =\operatorname{rank}_{a, b}^{\pi^{\prime \prime}}\left(B_{1}\right)+\operatorname{rank}_{a, b}^{\pi^{\prime \prime}}\left(B_{2}\right)+\operatorname{rank}_{a, b}^{\pi^{\prime \prime}}\left(B_{3}\right) \\
& =\left\lceil(j-i) \frac{a}{b}\right\rceil+\left\lceil(k-j) \frac{a}{b}\right]+\operatorname{rank}^{\pi^{\prime \prime}}\left(B_{3}\right) \\
& =\left\lceil(j-i) \frac{a}{b}\right\rceil+\left\lceil(k-j) \frac{a}{b}\right\rceil+a-\left\lceil(k-i) \frac{a}{b}\right\rceil \\
& >a+\left\lceil(j-i) \frac{a}{b}\right\rceil-(j-i) \frac{a}{b} \\
& >a,
\end{aligned}
$$

which is a contradiction. We conclude that $\pi^{\prime \prime}$ does not have a valid $a, b$-ranking.

As an application of Proposition 3.15, we get that the set $\mathrm{NC}(a, b)$ carries an action of not just the rotation operator rot, but the full dihedral group $\langle$ rot, $r f n\rangle$. This gives another application of $a, b$-ranks.

Corollary 3.16. Let $a<b$ be coprime. The set $\mathrm{NC}(a, b)$ of $a, b$-noncrossing partitions is closed under the action of the dihedral group 〈rot, $\mathrm{rfn}\rangle$.

Proof. It is enough to show that $\mathrm{NC}(a, b)$ is closed under $\mathrm{rfn}$. For any noncrossing partition $\pi \in \mathrm{NC}(b-1)$ and any block $B$ of $\pi$, we have

$$
\operatorname{rank}_{a, b}^{\pi}(B)=\operatorname{rank}_{a, b}^{\mathrm{rfn}(\pi)}(\operatorname{rfn}(B)) .
$$


It follows that every element in the rotation orbit of $\pi$ has a valid $a, b$-ranking if and only if every element in the rotation orbit of $\operatorname{rfn}(\pi)$ has a valid $a, b$-ranking.

\section{Modified rank sequences}

In this section we will study rational noncrossing partitions which have nontrivial rotational symmetry. Our key tool will the the theory of $a, b$-rank developed in Section 3. We fix the following

Notation. For the remainder of this section, let $a<b$ be coprime positive integers. Let $d \mid(b-1)$ be a divisor with $1 \leqslant d<b-1$. Let $q:=\frac{b-1}{d}$. Let

$$
\mathrm{NC}_{d}(a, b):=\left\{\pi \in \mathrm{NC}(a, b): \operatorname{rot}^{d}(\pi)=\pi\right\}
$$

denote the set of a,b-noncrossing partitions which are fixed by $\operatorname{rot}^{d}$.

The numerology of $\mathrm{NC}_{d}(a, b)$ will turn out to be somewhat simpler than that of $\mathrm{NC}(a, b)$ itself. We begin by defining a modified version of the rank sequence which is well suited to studying partitions with rotational symmetry.

Let $\pi \in \mathrm{NC}_{d}(a, b)$. The fact that $\pi$ is noncrossing implies that $\pi$ has at most one block $B_{0}$ which satisfies $\operatorname{rot}^{d}\left(B_{0}\right)=B_{0}$. If such a block $B_{0}$ exists, it is called central. Moreover, the cyclic group $\mathbb{Z}_{q}=\left\langle\operatorname{rot}^{d}\right\rangle$ acts freely on the non-central blocks of $\pi$. A non-central block $B$ of $\pi$ is called wrapping if the interval $[\min (B), \max (B)]$ contains every block in the $\left\langle\operatorname{rot}^{d}\right\rangle$-orbit of $B$. Any $\left\langle\operatorname{rot}^{d}\right\rangle$-orbit of blocks has at most one wrapping block.

Definition 4.1. Let $\pi \in \mathrm{NC}_{d}(a, b)$. The $d$-modified rank sequence of $\pi$ is the length $d$ sequence $S_{d}(\pi)=\left(s_{1}, \ldots, s_{d}\right)$ of nonnegative integers defined by

$$
s_{i}:= \begin{cases}\operatorname{rank}_{a, b}^{\pi}(B) & \text { if } i=\min (B) \text { for some non-central, non-wrapping block } B \in \pi, \\ 0 & \text { otherwise. }\end{cases}
$$

For example, suppose that $(a, b)=(4,9), d=4$, and $\pi=\{\{1,8\},\{2,3,6,7\},\{4,5\}\} \in$ $\mathrm{NC}_{4}(4,9)$. The block $\{1,8\}$ of $\pi$ is wrapping and the block $\{2,3,6,7\}$ of $\pi$ is central. Since the 4,9-rank of $\{4,5\}$ is 1 , the 4-modified rank sequence of $\pi$ is $S_{4}(\pi)=(0,0,0,1)$.

The $d$-modified rank sequence $S_{d}(\pi)$ is like the ordinary rank sequence $R(\pi)$, but we only consider the indices in $[d]$ rather than in $[b-1]$ and we only keep track of the ranks of blocks which are neither central nor wrapping. It is true, but not obvious at this point, that a set partition $\pi \in \mathrm{NC}_{d}(a, b)$ is determined by $S_{d}(\pi)$.

Our first lemma states that the assignment $\pi \mapsto S_{d}(\pi)$ commutes with the action of rotation.

Lemma 4.2. Let $\pi \in \mathrm{NC}_{d}(a, b)$ and let $S_{d}(\pi)=\left(s_{1}, s_{2}, \ldots, s_{d}\right)$ be the d-modified rank sequence of $\pi$. We have

$$
S_{d}(\operatorname{rot}(\pi))=\operatorname{rot}\left(S_{d}(\pi)\right),
$$

where $\operatorname{rot}\left(s_{1}, s_{2}, \ldots, s_{d}\right)=\left(s_{d}, s_{1}, \ldots, s_{d-1}\right)$ is the rotation operator on sequences. 
Proof. Let $S_{d}(\operatorname{rot}(\pi))=\left(s_{1}^{\prime}, s_{2}^{\prime}, \ldots, s_{d}^{\prime}\right)$ be the $d$-modified rank sequence of $\operatorname{rot}(\pi)$ and let $1 \leqslant i \leqslant q$. We show that $s_{i}^{\prime}=s_{i-1}$, where subscripts are interpreted modulo $d$. We will make free use of Proposition 3.10, which implies that $\operatorname{rank}_{a, b}^{\pi}(B)=\operatorname{rank}_{a, b}^{\operatorname{rot}(\pi)}(\operatorname{rot}(B))$ for any block $B \in \pi$.

Case 1: $2 \leqslant i \leqslant d$. Suppose $s_{i-1}>0$. Then $i-1=\min (B)$ for some non-central, nonwrapping block $B \in \pi$. Since $B$ is non-central and non-wrapping and $1 \leqslant \min (B) \leqslant d-1$, we know that $\operatorname{rot}(B)$ is also non-central and non-wrapping with $\min (\operatorname{rot}(B))=i$. We conclude that $s_{i}^{\prime}=s_{i-1}$.

Suppose $s_{i-1}=0$. If $i-1$ is not the minimum element of a block of $\pi$, then $i$ is not the minimum element of a block of $\operatorname{rot}(\pi)$, so that $s_{i}^{\prime}=0$. If $i-1=\min \left(B_{0}\right)$ for a central block $B_{0} \in \pi$, then $\operatorname{rot}\left(B_{0}\right)$ is a central block in $\operatorname{rot}(\pi)$ with $i \in \operatorname{rot}\left(B_{0}\right)$, so that $s_{i}^{\prime}=0$. If $i-1=\min (B)$ for a wrapping block $B \in \pi$, then the fact that $1 \leqslant \min (B) \leqslant d-1$ implies that either $i \neq \min (\operatorname{rot}(B))$ or $(\operatorname{rot}(B)$ is wrapping with $i \in \operatorname{rot}(B))$. In either situation, we get that $s_{i}^{\prime}=0$.

Case 2: $i=1$. Suppose $s_{d}>0$. Then $d=\min (B)$ for some non-central, non-wrapping block $B \in \pi$. Recalling that $\operatorname{rot}^{d}(\pi)=\pi$, it follows that $\operatorname{rot}^{d(q-1)+1}(B)$ is a non-central, non-wrapping block of $\operatorname{rot}(\pi)$ containing 1 . Thus, we get

$$
s_{1}^{\prime}=\operatorname{rank}_{a, b}^{\operatorname{rot}(\pi)}\left(\operatorname{rot}^{d(q-1)+1}(B)\right)=\operatorname{rank}_{a, b}^{\pi}(B)=s_{q} .
$$

Suppose $s_{d}=0$. If $d$ is contained in a central block of $\pi$, then 1 is contained in a central block of $\operatorname{rot}(\pi)$ and $s_{1}^{\prime}=0$. Since $\pi$ is noncrossing and $\operatorname{rot}^{d}(\pi)=\pi$, the index $q$ cannot be contained in a wrapping block of $\pi$. If $d \in B$ for some block $B \in \pi$ which is non-central and non-wrapping, we must have that $d \neq \min (B)$. Since $\pi$ is noncrossing with $\operatorname{rot}^{d}(\pi)=\pi$, it follows that $\operatorname{rot}(B)$ is wrapping and $1 \in \operatorname{rot}(B)$, so that $s_{1}^{\prime}=0$.

What sequences $\left(s_{1}, \ldots, s_{d}\right)$ of nonnegative integers arise as $d$-modified rank sequences of partitions in $\mathrm{NC}_{d}(a, b)$ ? If $\pi \in \mathrm{NC}_{d}(a, b)$ and $S_{d}(\pi)=\left(s_{1}, \ldots, s_{d}\right)$ is the $d$-modified rank sequence of $\pi$, we claim that

$$
q\left(s_{1}+\cdots+s_{d}\right)=\sum_{B} \operatorname{rank}_{a, b}^{\pi}(B)
$$

where the sum is over all non-central blocks $B \in \pi$. Indeed, each $q$-element orbit of noncentral blocks contributes the rank of one of its constituents precisely once to the nonzero terms in $S_{d}(\pi)$. By Proposition 3.15,

$$
s_{1}+\cdots+s_{d} \leqslant \frac{a}{q},
$$

with equality if and only if $\pi$ does not have a central block. (Unless $q \mid a$, the partition $\pi$ necessarily has a central block.)

We call a length $d$ sequence $\left(s_{1}, \ldots, s_{d}\right)$ of nonnegative integers good if we have the inequality $s_{1}+\cdots+s_{d} \leqslant \frac{a}{q}$. The goal for the remainder of this section is to show that the map

$$
S_{d}: \mathrm{NC}_{d}(a, b) \longrightarrow\left\{\text { good sequences }\left(s_{1}, \ldots, s_{d}\right)\right\}
$$


is a bijection. Since good sequences are easily enumerated, this will give us information about $\mathrm{NC}_{d}(a, b)$. The strategy is to isolate nice subsets of $\mathrm{NC}_{d}(a, b)$ and the set of good sequences which contain at least one representative from every rotation orbit, show that these subsets are in bijection under $S_{d}$, and apply Lemma 4.2.

Definition 4.3. A set partition $\pi \in \mathrm{NC}_{d}(a, b)$ is noble if $\pi$ does not contain any wrapping blocks and, if $\pi$ contains a central block $B_{0}$, we have that $1 \in B_{0}$.

For example, consider the case $(a, b)=(6,7)$ and $d=3$. Then

$$
\pi=\{\{1\},\{2,3,5,6\},\{4\}\} \in \mathrm{NC}_{3}(6,7)
$$

is not a noble partition because 1 is not contained in the central block. On the other hand, both of the rotations $\{\{1,3,4,6\},\{2\},\{5\}\}$ and $\{\{1,2,4,5\},\{3\},\{6\}\}$ of $\pi$ are noble partitions. Also, if $(a, b)=(6,7)$ and $d=2$ the partition $\sigma=\{\{1,6\},\{2,3\},\{4,5\}\} \in$ $\mathrm{NC}_{2}(6,7)$ is not a noble partition because the block $\{1,6\}$ is wrapping. However, the rotation $\{\{1,2\},\{3,4\},\{5,6\}\}$ of $\sigma$ is a noble partition. In general, we have the following observation.

Observation 4.4. Every $\langle$ rot $\rangle$-orbit in $\mathrm{NC}_{d}(a, b)$ contains at least one noble partition.

The notion of nobility for sequences involves an intermediate definition. Let $s=$ $\left(s_{1}, \ldots, s_{d}\right)$ be a good sequence. We call $s$ very good if $s_{1}+\cdots+s_{d}=\frac{a}{q}$ or $s_{1}=0$.

We define a map

$$
L:\{\text { very good sequences }\} \longrightarrow \text { lattice paths from }(0,0) \text { to }(b, a)\}
$$

as follows. If $s=\left(s_{1}, \ldots, s_{q}\right)$ is a very good sequence, let $L(s)$ be the lattice path obtained by taking the $q$-fold concatenation of the path $N^{s_{1}} E \ldots N^{s_{d}} E$, adding a terminal east step, and if $s_{1}+\cdots+s_{d}<\frac{a}{q}$ adding an initial vertical run of size $c:=a-q\left(s_{1}+\cdots+s_{d}\right)$. In symbols,

$$
L(s):= \begin{cases}\left(N^{s_{1}} E \ldots N^{s_{d}} E\right)^{q} E & \text { if } s_{1}+\cdots+s_{d}=\frac{a}{q}, \\ \left(N^{c} E N^{s_{2}} E \ldots N^{s_{d}} E\right)\left(N^{s_{1}} E \ldots N^{s_{d}} E\right)^{q-1} E & \text { if } s_{1}+\cdots+s_{d}<\frac{a}{q} .\end{cases}
$$

Since $s$ is assumed to be very good, we get that $L(s)$ ends at $(b, a)$ so that the map $L$ is well defined. We will refer to the subpaths $L_{1}, \ldots, L_{q}$ defined by the above factorization of $L(s)$ as the segments of $L(s)$, so that $L(s)=L_{1} \cdots L_{q} E$.

If $s$ is a very good sequence, the lattice path $L(s)$ is typically not an $a, b$-Dyck path. For example, if $(a, b)=(4,7), d=3$, and $s=\left(s_{1}, s_{2}, s_{3}\right)=(0,1,1)$, we have

$$
L(s)=\left(N^{0} E N^{1} E N^{1} E\right)\left(N^{0} E N^{1} E N^{1} E\right) E,
$$

which is not a 4,7-Dyck path.

Definition 4.5. A very good sequence $s=\left(s_{1}, \ldots, s_{d}\right)$ is noble if $L(s)$ is an a,b-Dyck path. 
When $(a, b)=(4,7)$ and $d=3$, the above example shows that $(0,1,1)$ is not a noble sequence. On the other hand, the rotation $(1,1,0)$ of $(0,1,1)$ is a noble sequence. In general, we have the following analog of Observation 4.4 for good sequences.

Lemma 4.6. Every good sequence is 〈rot〉-conjugate to at least one noble sequence.

Proof. Let $\left(s_{1}, \ldots, s_{d}\right)$ be a good sequence and set $c=a-q\left(s_{1}+\cdots+s_{d}\right)$.

Case 1: $c=a$. In this case $\left(s_{1}, \ldots, s_{d}\right)$ is the zero sequence $(0,0, \ldots, 0)$ and is trivially noble.

Case 2: $0<c<a$. The argument we present here is a modification of the argument used to prove the Cycle Lemma.

Consider the lattice path $L$ which starts at the origin and ends at $\left(2 d, 2\left(s_{1}+\cdots+s_{d}\right)\right)$ given by

$$
L=\left(N^{s_{1}} E \ldots N^{s_{d}} E\right)\left(N^{s_{1}} E \ldots N^{s_{d}} E\right) .
$$

As is common in rational Catalan theory, we label the lattice points $P$ on $L$ with integers $w(P)$ as follows. The origin is labeled 0 . Reading $L$ from left to right, if $P$ and $P^{\prime}$ are consecutive lattice points, we set $w\left(P^{\prime}\right)=w(P)-a$ if $P^{\prime}$ is connected to $P$ with an $E$-step and $w\left(P^{\prime}\right)=w(P)+b$ if $P^{\prime}$ is connected to $P$ with an $N$-step.

For example, suppose that $(a, b)=(11,13), d=4$, and $\left(s_{1}, s_{2}, s_{3}, s_{4}\right)=(1,0,2,0)$. We have $q=\frac{13}{4}=3$ and $c=11-3(1+0+2+0)=2$. The lattice path $L$, together with the labels of its lattice points, is as follows.

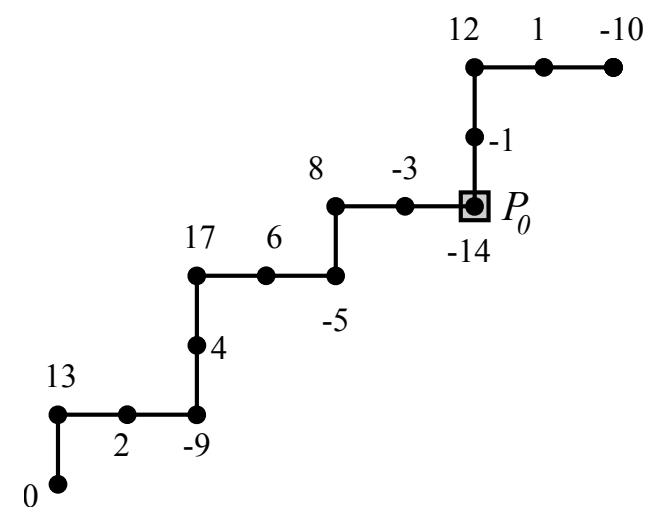

By coprimality, there exists a unique lattice point on $L$ of minimal weight. Let $P_{0}$ be this lattice point; in the above example, we have $w\left(P_{0}\right)=-14$. We claim that $P_{0}$ occurs after a pair of consecutive east steps $E E$. Indeed, since $0<c<a$, we know that the weight of the terminal lattice point of $L$ is negative (in the above example, -10 ), so that $P_{0}$ is not the origin. If $P_{0}$ did not occur after a pair of consecutive east steps, by the minimality of $w\left(P_{0}\right)$, we have that $P_{0}$ occurs after a pair $N E$. But since $a<b$ the lattice point $P_{0}^{\prime}$ occurring at the beginning of this $N E$-sequence satisfies $w\left(P_{0}^{\prime}\right)=w\left(P_{0}\right)+a-b<w\left(P_{0}\right)$, a contradiction. Therefore, the lattice point $P_{0}$ does indeed occur after a pair of consecutive east steps $E E$.

The minimality of $w\left(P_{0}\right)$ and the fact that $\left(s_{1}, \ldots, s_{d}\right) \neq(0, \ldots, 0)$ mean that $P_{0}$ is immediately followed by a nonempty vertical run $N^{s_{i}}$ for some $1 \leqslant i \leqslant d$. Since $P_{0}$ is 
preceded by a pair $E E$, we get that $i \geqslant 2$ and $s_{i-1}=0$. In the above example, we have $i=3$. Since $s_{i-1}=0$, the rotated sequence $\left(s_{i-1}, s_{i}, \ldots, s_{d}, s_{1}, s_{2}, \ldots, s_{i-2}\right)$ is very good. The lattice path $L\left(s_{i-1}, s_{i}, \ldots, s_{d}, s_{1}, s_{2}, \ldots, s_{i-2}\right)$ is therefore well defined.

In the above example, we have $\left(s_{i-1}, s_{i}, \ldots, s_{d}, s_{1}, s_{2}, \ldots, s_{i-2}\right)=(0,2,0,1)$ and the lattice path $L(0,2,0,1)$ is shown in the proof of Lemma 4.7 below. The segmentation $L(s)=L_{1} \ldots L_{q} E=L_{1} L_{2} L_{3} E$ is shown with vertical hash marks. Observe that $L(s)$ is an $a, b$-Dyck path in this case.

We claim that the lattice path $L\left(s_{i-1}, s_{i}, \ldots, s_{d}, s_{1}, s_{2}, \ldots, s_{i-2}\right)$ is always an $a, b$-Dyck path, so that the rotation $\left(s_{i-1}, s_{i}, \ldots, s_{d}, s_{1}, s_{2}, \ldots, s_{i-2}\right)$ is a noble sequence. Indeed, consider the segmentation $L(s)=L_{1} \ldots L_{q} E$. The segments $L_{1}, \ldots, L_{q}$ will be progressively further east, so it is enough to show that the final segment $L_{q}$ stays west of the line $y=x$. By construction, the segment $L_{q}$ starts with a single east step, hits the copy of the point $P_{0}$, then then has a nonempty vertical run, and eventually ends at the point $(b-1, a)$. Since $\left(s_{1}, \ldots, s_{d}\right)$ is a good sequence, we know that $L_{q}$ starts at a lattice point to the west of the line $y=\frac{a}{b}(x+1)$. The minimality of $w\left(P_{0}\right)$ forces $L_{q}$ to remain west of the line $y=\frac{a}{b} x$.

Case 3: $c=0$. This is a special case of the Cycle Lemma; the argument is very similar to Case 2 and is only sketched. We again consider the lattice path $L$ given by $L=\left(N^{s_{1}} E \ldots N^{s_{d}} E\right)\left(N^{s_{1}} E \ldots N^{s_{d}} E\right)$ and assign weights $w(P)$ to the lattice points $P$ on $L$ as before. There exists a unique lattice point $P_{0}$ on $L$ with minimal weight. The lattice point $P_{0}$ necessarily occurs before a nonempty vertical run $N^{s_{i}}$ for some $1 \leqslant i \leqslant d$. The rotation $\left(s_{i}, s_{i+1}, \ldots, s_{d}, s_{1}, s_{2}, \ldots, s_{i-1}\right)$ of $s$ is a noble sequence.

Given any noble sequence $s$, we may consider the $a, b$-noncrossing partition $\pi(L(s))$ corresponding to the Dyck path $L(s)$. We prove that $\pi(L(s))$ is $\operatorname{rot}^{d}$-invariant and noble.

Lemma 4.7. Suppose that $s=\left(s_{1}, \ldots, s_{d}\right)$ is a noble sequence Then $\pi:=\pi(L(s))$ is a noble a,b-noncrossing partition (and in particular $\operatorname{rot}^{d}(\pi)=\pi$ ) with $S_{d}(\pi)=s$.

Proof. Recall the visibility bijection between blocks of $\pi$ and nonempty vertical runs in $L(s)$. Let $c=a-q\left(s_{1}+\cdots+s_{d}\right)$. The argument depends on whether $c>0$ or $c=0$.

Case 1: $c>0$. We consider the segmentation of $L(s)$ given by

$$
L(s)=L_{1} L_{2} \ldots L_{q} E,
$$

where $L_{1}=N^{c} E N^{s_{2}} E \ldots N^{s_{d}} E$ and $L_{j}=N^{s_{1}} E N^{s_{2}} E \ldots N^{s_{d}} E$ for $2 \leqslant j \leqslant q$.

As an example of this case, consider $(a, b)=(11,13), d=4, s=(0,2,0,1)$. Then $s$ is a noble sequence. The 11,13-Dyck path $L(s)=L_{1} L_{2} L_{3} E$ is shown below, where vertical hash marks separate the segments $L_{1}, L_{2}$, and $L_{3}$. The valley lasers from $L(s)$ are as shown. For visibility, we suppress the labels on the interior lattice points. 


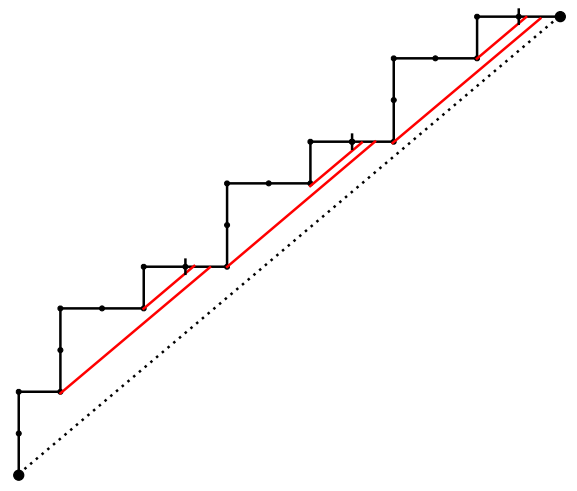

Since $s$ is noble and $c>0$, we have $s_{1}=0$. Fix any index $2 \leqslant i \leqslant d$ such that $s_{i}>0$ and another index $1 \leqslant j \leqslant q-1$. Both of the segments $L_{j}$ and $L_{j+1}$ of the $a, b$-Dyck path $L(s)$ contain a copy of the nonempty vertical run $N^{s_{i}}$. If $P_{0}$ and $P_{1}$ are the valleys at the bottom of these vertical runs in $L_{j}$ and $L_{j+1}$, respectively, the lasers $\ell\left(P_{0}\right)$ and $\ell\left(P_{1}\right)$ fired from $P_{0}$ and $P_{1}$ are (rigid) translations of the same line segment. In particular, the block visible from the copy of $N^{s_{i}}$ in $L_{j+1}$ is the image of the block visible from the copy of $N^{s_{i}}$ in $L_{j}$ by the operator $\operatorname{rot}^{d}$. Moreover, the fact that $s_{1}=0$ means that neither of these blocks contain the index 1 .

In the above example, we have three segments $\left(L_{1}, L_{2}\right.$, and $\left.L_{3}\right)$ with $s_{i}>0$ for $i=2,4$. The blocks visible from the copies of $N^{s_{2}}=N^{2}$ in $L_{1}, L_{2}$, and $L_{3}$ are $\{2,3\},\{6,7\}$, and $\{10,11\}$, respectively. The blocks visible from the copies of $N^{s_{4}}=N^{1}$ in $L_{1}, L_{2}$, and $L_{3}$ are $\{4\},\{8\}$, and $\{12\}$, respectively. The block visible from the initial vertical run is $\{1,5,9\}$, which is central.

In general, we conclude that the set of blocks of $\pi$ not containing 1 is stable under the action of $\operatorname{rot}^{d}$, so that the block of $\pi$ containing 1 must be central. We get that $\pi \in \mathrm{NC}_{d}(a, b)$. Since $\pi \in \mathrm{NC}_{d}(a, b)$ has a central block containing 1 , the partition $\pi$ contains no wrapping blocks. It follows that $\pi$ in noble and $S_{d}(\pi)=s$.

Case 2: $c=0$. In this case, $\pi$ does not contain a central block. We again consider the segmentation $L(s)=L_{1} L_{2} \ldots L_{q} E$ as in Case 1. Here $L_{j}=N^{s_{1}} E N^{s_{2}} E \ldots N^{s_{d}} E$ for $1 \leqslant j \leqslant q$.

As an example of this case, consider $(a, b)=(9,13), d=4$, and $s=(1,2,0,0)$. Then $s$ is a noble sequence. The 9,13 -Dyck path $L(s)$ is shown below, with diagonal hash marks denoting the segmentation $L(s)=L_{1} L_{2} L_{3} E$. The valley lasers of $L(s)$ are shown, and the interior lattice point labels are suppressed.

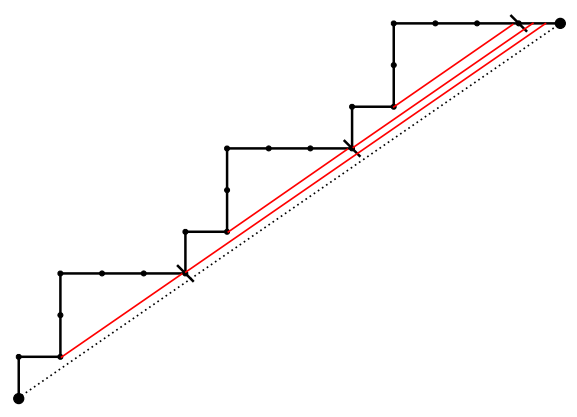


Let $1 \leqslant i \leqslant d$ be an index such that $s_{i}>0$ and consider any two consecutive segments $L_{j}$ and $L_{j+1}$ of $L(s)$. The lasers fired from the valleys below the copies of $N^{s_{i}}$ both $L_{j}$ and $L_{j+1}$ are either

- translates of each other, or

- both hit the Dyck path $L(s)=L_{1} \ldots L_{q} E$ on its terminal east step.

In the above example, we see that the lasers fired from each copy of $N^{s_{2}}=N^{2}$ are translates of one another, and the lasers fired from each copy of $N^{s_{1}}=N^{1}$ hit $L(s)$ on its terminal east step. This implies that the blocks corresponding of these vertical runs are $\left\langle\operatorname{rot}^{d}\right\rangle$-conjugate, so that $\pi \in \mathrm{NC}_{d}(a, b)$.

Moreover, since $c=0$ the laser fired from any copy of the (nonempty) vertical run $N^{s_{1}}$ in $L(s)=L_{1} \ldots L_{q} E$ must hit $L(s)$ on its terminal east step. This implies that $\pi$ has no wrapping blocks, so that $\pi$ is noble and $S_{d}(\pi)=s$.

We show that nobility for sequences and nobility for partitions coincide.

Lemma 4.8. Suppose that $\pi \in \mathrm{NC}_{d}(a, b)$. Then $\pi$ is noble if and only if $S_{d}(\pi)$ is noble.

Proof. Write $S_{d}(\pi)=\left(s_{1}, \ldots, s_{d}\right)$ and set $c=a-q\left(s_{1}+\cdots+s_{d}\right)$.

$(\Rightarrow)$ Suppose $\pi$ is noble. Since $\pi$ contains no wrapping blocks, the rank sequence $R(\pi)$ is given by the formula

$$
R(\pi)= \begin{cases}\left(s_{1}, \ldots, s_{d}, s_{1}, \ldots, s_{d}, \ldots, s_{1}, \ldots, s_{d}\right) & \text { if } s_{1}+\cdots+s_{d}=\frac{a}{q} \\ \left(c, \ldots, s_{d}, s_{1}, \ldots, s_{d}, \ldots, s_{1}, \ldots, s_{d}\right) & \text { if } s_{1}+\cdots+s_{d}<\frac{a}{q}\end{cases}
$$

If $c>0$, since 1 is contained in the central block of $\pi$ we get $s_{1}=0$, so that $S_{d}(\pi)$ is very good. By Proposition 3.8, we get that $L(s)$ is an $a, b$-Dyck path, so that $S_{d}(\pi)$ is noble.

$(\Leftarrow)$ Suppose $S_{d}(\pi)$ is noble. We claim that $\pi$ contains no wrapping blocks. Working towards a contradiction, assume that $\pi$ contains at least one wrapping block and choose a wrapping block $B$ of $\pi$ such that the interval $[\min (B), \max (B)]$ is maximal under containment.

If $1 \in B$, then we would have $s_{1}=0$ since $B$ is wrapping, making the first step of the path $L\left(S_{d}(\pi)\right)$ an east step. This contradicts the nobility of $S_{d}(\pi)$. We conclude that $1 \notin B$.

Since $1 \notin B$, the interval $[1, \min (B)-1]$ is nonempty. By our choice of $B$, we have that $[1, \min (B)-1]$ is a union blocks of $\pi$, none of which are central or wrapping. This means that the first $\min (B)-1$ terms in the $d$-modified rank sequence $S_{d}(\pi)$ coincide with the first $\min (B)-1$ terms in the ordinary rank sequence $R(\pi)$.

By Proposition 3.8, the $a, b$-Dyck path corresponding to $\pi$ starts at the origin with the subpath $N^{s_{1}} E N^{s_{2}} E \ldots N^{s_{\min (B)-1}} E$. Since $[1, \min (B)-1]$ is a union of blocks of $\pi$, Corollary 3.9 guarantees that this subpath ends at the point $\left(\min (B)-1,\left\lceil\frac{a}{b}(\min (B)-1)\right\rceil\right)$. It follows that the subpath $\left(N^{s_{1}} E N^{s_{2}} E \ldots N^{s_{\min (B)-1}} E\right) E$ obtained by appending a single east step crosses the diagonal $y=\frac{a}{b} x$. But since $B$ is wrapping, we have $s_{\min (B)}=0$, 
so that this is an initial subpath of $L\left(S_{d}(\pi)\right)$, so that $L\left(S_{d}(\pi)\right)$ is not an $a, b$-Dyck path. This contradicts the nobility of $S_{d}(\pi)$. We conclude that $\pi$ contains no wrapping blocks.

Suppose that $\pi$ contains a central block $B_{0}$. We need to prove that $1 \in B_{0}$. If $1 \notin B_{0}$, the fact that $\pi$ does not contain wrapping blocks implies that $\left[1, \min \left(B_{0}\right)-1\right]$ is a union of non-wrapping, non-central blocks of $\pi$. The first $\min \left(B_{0}\right)-1$ terms of the $d$-modifed rank sequence $S_{d}(\pi)$ coincide with the corresponding terms of the ordinary rank sequence $R(\pi)$. The same reasoning as in the last paragraph implies that the lattice path $L\left(S_{d}(\pi)\right)$ contains the point $\left(\min \left(B_{0}\right)-1,\left\lceil\frac{a}{b}\left(\min \left(B_{0}\right)-1\right)\right\rceil\right)$. However, since $B_{0}$ is central, we have that $s_{\min \left(B_{0}\right)}=0$, so that the lattice path $L\left(S_{d}(\pi)\right)$ has an east step originating from this lattice point. But this means that $L\left(S_{d}(\pi)\right)$ is not an $a$, b-Dyck path, contradicting the nobility of $S_{d}(\pi)$. We conclude that $1 \in B_{0}$, and that $\pi$ is a noble partition.

We have the lemmata we need to prove that the map $S_{d}$ is a bijection. In particular, partitions in $\mathrm{NC}_{d}(a, b)$ are determined by their $d$-modified rank sequences.

Proposition 4.9. The map $S_{d}: \mathrm{NC}_{d}(a, b) \longrightarrow\left\{\right.$ good sequences $\left.\left(s_{1}, \ldots, s_{d}\right)\right\}$ is a bijection which commutes with the action of rotation.

Proof. By Lemma 4.2, we know that $S_{d}$ commutes with rotation. If $s$ is a noble sequence, Lemma 4.7 shows that $S_{d}^{-1}(s)$ is nonempty, and Lemma 4.6 shows that $S_{d}$ is surjective.

To see that $S_{d}$ is injective, let $s$ be a noble sequence and suppose $\pi \in \mathrm{NC}_{d}(a, b)$ satisfies $S_{d}(\pi)=s=\left(s_{1}, \ldots, s_{d}\right)$. By Lemma 4.8 , the partition $\pi$ is noble. The rank sequence $R(\pi)$ is therefore

$$
R(\pi)= \begin{cases}\left(s_{1}, \ldots, s_{d}, s_{1}, \ldots, s_{d}, \ldots, s_{1}, \ldots, s_{d}\right) & \text { if } s_{1}+\cdots+s_{d}=\frac{a}{q} \\ \left(c, \ldots, s_{d}, s_{1}, \ldots, s_{d}, \ldots, s_{1}, \ldots, s_{d}\right) & \text { if } s_{1}+\cdots+s_{d}<\frac{a}{q}\end{cases}
$$

where $c=a-q\left(s_{1}+\cdots+s_{d}\right)$. By Proposition 3.8, any $a, b$-noncrossing partition is determined by its rank sequence. We conclude that $\left|S_{d}^{-1}(s)\right|=1$. By Observation 4.4 and Lemma 4.6, together with the fact that $S_{d}$ commutes with rot (Lemma 4.2), we conclude that $S_{d}$ is a bijection.

Since every nonzero entry in a $d$-modified rank sequence $S_{d}(\pi)=\left(s_{1}, \ldots, s_{d}\right)$ corresponds to a $\left\langle\operatorname{rot}^{d}\right\rangle$-orbit of non-central blocks of $\pi$ of that rank, the following result follows immediately from Proposition 4.9 .

Corollary 4.10. Let $a<b$ be coprime positive integers and let $d \mid(b-1)$ be a divisor with $1 \leqslant d<b-1$. Let $m_{1}, \ldots, m_{a}$ be nonnegative integers which satisfy $\frac{b-1}{d}\left(m_{1}+2 m_{2} \cdots+\right.$ $\left.a m_{a}\right) \leqslant a$. Write $m:=m_{1}+m_{2}+\cdots+m_{a}$.

The number of a,b-noncrossing partitions which are invariant under $\operatorname{rot}^{d}$ and have $m_{i}$ orbits of non-central blocks of a,b-rank $i$ under the action of $\left\langle\operatorname{rot}^{d}\right\rangle$ is the multinomial coefficient

$$
\left(\begin{array}{c}
d \\
m_{1}, m_{2}, \ldots, m_{a}, d-m
\end{array}\right)
$$


The Fuss-Catalan case $b \equiv 1(\bmod a)$ of Corollary 4.10 is equivalent to a result of Athanasiadis [5, Theorem 2.3].

We can also consider counting partitions in $\mathrm{NC}_{d}(a, b)$ with a fixed number of noncentral block orbits (of any rank). By Proposition 4.9, this is equivalent to counting sequences $\left(s_{1}, \ldots, s_{d}\right)$ of nonnegative integers with bounded sum and a fixed number of nonzero entries.

Corollary 4.11. Let $a<b$ be coprime positive integers and let $d \mid(b-1)$ be a divisor with $1 \leqslant d<b-1$. Let $p$ be a nonnegative integer with $\frac{b-1}{d} p \leqslant a$.

The number of a,b-noncrossing partitions which are invariant under $\operatorname{rot}^{d}$, have a central block, and have $p$ orbits of non-central blocks under the action of $\left\langle\operatorname{rot}^{d}\right\rangle$ is

$$
\left(\begin{array}{l}
d \\
p
\end{array}\right)\left(\begin{array}{c}
\left\lfloor\frac{a d}{b-1}\right\rfloor-1 \\
p
\end{array}\right)
$$

The number of $a, b$-noncrossing partitions which are invariant under $\operatorname{rot}^{d}$, do not have a central block, and have $p$ orbits of non-central blocks under the action of $\left\langle\operatorname{rot}^{d}\right\rangle$ is

$$
\begin{cases}\left(\begin{array}{l}
d \\
p
\end{array}\right)\left(\begin{array}{c}
\frac{a d}{b-1}-1 \\
p-1
\end{array}\right) & \text { if } \frac{b-1}{d} \mid a, \\
0 & \text { if } \frac{b-1}{d} \nmid a .\end{cases}
$$

Proof. For the first part, we choose $p$ entries in the sequence $\left(s_{1}, \ldots, s_{d}\right)$ to be nonzero. Then, we assign positive values to these $p$ entries in such a way that their sum is $<\left\lfloor\frac{a d}{b-1}\right\rfloor$. The second part is similar.

Finally, we can consider the problem of counting $\mathrm{NC}_{d}(a, b)$ itself. By Proposition 4.9, this corresponds to counting sequences $\left(s_{1}, \ldots, s_{d}\right)$ of nonnegative integers which satisfy $s_{1}+\cdots+s_{d} \leqslant\left\lfloor\frac{a d}{b-1}\right\rfloor$.

Corollary 4.12. Let $a<b$ be coprime positive integers and let $d \mid(b-1)$ be a divisor with $1 \leqslant d<b-1$.

The number of $a, b$-noncrossing partitions which are invariant under $\operatorname{rot}^{d}$ is

$$
\left(\begin{array}{c}
\left\lfloor\frac{a d}{b-1}\right\rfloor+d \\
d
\end{array}\right)
$$

The Fuss-Catalan cases $b \equiv 1(\bmod a)$ of Corollaries 4.11 and 4.12 are results of Reiner [8, Propositions 6 and 7].

\section{Cyclic sieving}

Let $X$ be a finite set, let $C=\langle c\rangle$ be a finite cyclic group acting on $X$, let $X(q) \in \mathbb{N}[q]$ be a polynomial with nonnegative integer coefficients, and let $\zeta \in \mathbb{C}$ be a root of unity with multiplicative order $|C|$. The triple $(X, C, X(q))$ exhibits the cyclic sieving phenomenon 
$\left(C S P\right.$ ) if, for all $d \geqslant 0$, we have $X\left(\zeta^{d}\right)=\left|X^{c^{d}}\right|=\left|\left\{x \in X: c^{d} \cdot x=x\right\}\right|$ (see [10]). In this section we prove cyclic sieving results for the action of rotation on $\mathrm{NC}(a, b)$.

Our proofs will be 'brute force' and use direct root-of-unity evaluations of $q$-analogs. We will make frequent use of the following fact: If $x \equiv y(\bmod z)$, then

$$
\lim _{q \rightarrow e^{2 \pi i / z}} \frac{[x]_{q}}{[y]_{q}}= \begin{cases}\frac{x}{y} & \text { if } y \equiv 0 \bmod z, \\ 1 & \text { otherwise. }\end{cases}
$$

From this, we get the useful fact that

$$
\lim _{q \rightarrow e^{2 \pi i / z}} \frac{[n z]_{q} !}{[k z]_{q} !}=\left.\left(\begin{array}{l}
n \\
k
\end{array}\right)[n z-k z]_{q} !\right|_{q=e^{2 \pi i / z} .}
$$

Theorem 5.1. Let $a<b$ be coprime and let $\mathbf{r}=\left(r_{1}, r_{2}, \ldots, r_{a}\right)$ be a sequence of nonnegative integers satisfying $\sum_{i=1}^{a} i r_{i}=a$. Set $k:=\sum_{i=1}^{a} r_{i}$. Let $X$ be the set of $a, b$-noncrossing partitions of $[b-1]$ with $r_{1}$ blocks of rank $1, r_{2}$ blocks of rank $2, \ldots$, and $r_{a}$ blocks of rank a.

The triple $(X, C, X(q))$ exhibits the cyclic sieving phenomenon, where $C=\mathbb{Z}_{b-1}$ acts on $X$ by rotation and

$$
X(q)=\operatorname{Krew}_{q}(a, b, \mathbf{r})=\frac{[b-1] !_{q}}{\left[r_{1}\right] !_{q}\left[r_{2}\right] !_{q} \cdots\left[r_{a}\right] !_{q}[b-k] !_{q}}
$$

is the q-rational Kreweras number.

Proof. Reiner and Sommers proved that the $q$-Kreweras number $\operatorname{Krew}_{q}(a, b, \mathbf{r})$ is polynomial in $q$ with nonnegative integer coefficients using algebraic techniques [9]. No combinatorial proof of the polynomiality or the positivity of $\operatorname{Krew}_{q}(a, b, \mathbf{r})$ is known. That is, there is no known statistic on $a, b$-noncrossing partitions of type $\mathbf{r}$ whose generating function is $\operatorname{Krew}_{q}(a, b, \mathbf{r})$.

On the other hand, we have the following elementary proof that $\operatorname{Krew}_{q}(a, b, \mathbf{r})$ is a polynomial in $q$ with nonnegative coefficients. The authors are grateful to an anonymous referee for pointing it out and allowing us to reproduce it here.

We start by showing that the rational expression

$$
\operatorname{Krew}_{q}(a, b ; \mathbf{r})=\frac{1}{[k]_{q}}\left[\begin{array}{c}
r_{1}+r_{2}+\cdots+r_{a} \\
r_{1}, r_{2}, \ldots, r_{a}
\end{array}\right]_{q}\left[\begin{array}{c}
b-1 \\
k-1
\end{array}\right]_{q}
$$

is a polynomial in $q$. For any positive integer $n$, we have

$$
q^{n}-1=\prod_{d \mid n} \Phi_{d}(q),
$$

where $\Phi_{d}(q)$ is the $d^{\text {th }}$ cyclotomic polynomial in $q$. Combining Equations 16 and 17 yields

$$
\operatorname{Krew}_{q}(a, b ; \mathbf{r})=\prod_{d \geqslant 2} \Phi_{d}(q)^{e_{d}}
$$


where

$$
e_{d}=-\sum_{i=1}^{a}\left\lfloor\frac{r_{i}}{d}\right\rfloor+\left\lfloor\frac{b-1}{d}\right\rfloor-\left\lfloor\frac{b-k}{d}\right\rfloor .
$$

To prove that $\operatorname{Krew}_{q}(a, b ; \mathbf{r})$ is a polynomial in $q$, we must show that $e_{d} \geqslant 0$ for all $d$.

To see that $e_{d} \geqslant 0$, we observe that the equation defining $e_{d}$ can be written in two different ways. We have

$$
\begin{aligned}
e_{d} & =-\chi(d \mid k)+\left(\left\lfloor\frac{k}{d}\right\rfloor-\sum_{i=1}^{a}\left\lfloor\frac{r_{i}}{d}\right\rfloor\right)+\left(\left\lfloor\frac{b-1}{d}\right\rfloor-\left\lfloor\frac{b-k}{d}\right\rfloor-\left\lfloor\frac{k-1}{d}\right\rfloor\right) \\
& =-\chi(d \mid b)+\left(\left\lfloor\frac{k}{d}\right\rfloor-\sum_{i=1}^{a}\left\lfloor\frac{r_{i}}{d}\right\rfloor\right)+\left(\left\lfloor\frac{b}{d}\right\rfloor-\left\lfloor\frac{b-k}{d}\right\rfloor-\left\lfloor\frac{k}{d}\right\rfloor\right),
\end{aligned}
$$

where for any statement $\mathcal{S}$ we set $\chi(\mathcal{S})=1$ if $\mathcal{S}$ is true and $\chi(\mathcal{S})=0$ if $\mathcal{S}$ is false. The terms in the second parentheses of both right-hand sides are obviously nonnegative. Since $r_{1}+r_{2}+\cdots+r_{a}=k$, the terms in the first parentheses of both right-hand sides are also nonnegative. Thus, unless $d \mid k$ and $d \mid b$, we have $e_{d} \geqslant 0$.

We therefore assume that $d \mid k$ and $d \mid b$. If $d \mid r_{i}$ for all $1 \leqslant i \leqslant a$, then the relation $r_{1}+2 r_{2}+\cdots+a r_{a}=a$ forces $d \mid a$, which contradicts the coprimality of $a$ and $b$. Thus, there exists $1 \leqslant i_{0} \leqslant a$ such that $d \nmid r_{i_{0}}$, meaning that $\frac{r_{i_{0}}}{d}>\left\lfloor\frac{r_{i_{0}}}{d}\right\rfloor$, and hence

$$
\left\lfloor\frac{k}{d}\right\rfloor-\sum_{i=1}^{a}\left\lfloor\frac{r_{i}}{d}\right\rfloor \geqslant 1 .
$$

Either of the right-hand sides 19 or 20 implies that

$$
e_{d} \geqslant-1+1+0=0
$$

as desired. We conclude that $\operatorname{Krew}_{q}(a, b, \mathbf{r})$ is a polynomial in $q$ with integer coefficients.

It remains to show that the polynomial $\operatorname{Krew}_{q}(a, b, \mathbf{r})$ has nonnegative coefficients. To see this, observe that the product

$$
[k]_{q} \times \operatorname{Krew}_{q}(a, b, \mathbf{r})=\left[\begin{array}{c}
r_{1}+r_{2}+\cdots+r_{a} \\
r_{1}, r_{2}, \ldots, r_{a}
\end{array}\right]_{q}\left[\begin{array}{c}
b-1 \\
k-1
\end{array}\right]_{q}
$$

is a unimodal and reciprocal polynomial with nonnegative coefficients (since it can be written as a product of $q$-binomial coefficients). Since we know that $\operatorname{Krew}_{q}(a, b, \mathbf{r})$ is a polynomial in $q$ with integer coefficients, [1, Proposition 10.1 (iii)] applies and $\operatorname{Krew}_{q}(a, b, \mathbf{r}$ ) has nonnegative coefficients (see also the discussion before [10, Corollary 10.4]).

Let $\zeta=e^{\frac{2 \pi i}{b-1}}$ and let $d \mid(b-1)$ with $1 \leqslant d<b-1$. Write $t=\frac{b-1}{d}$. We have that $X\left(\zeta^{d}\right)=0$ unless at $t \mid r_{i}$ for all but at most one $1 \leqslant i \leqslant a$, and that $r_{i_{0}} \equiv 1(\bmod t)$ if $t \nmid r_{i_{0}}$. If the sequence $\mathbf{r}$ satisfies the condition of the last sentence, define a new sequence $\left(m_{1}, \ldots, m_{a}\right)$ by $m_{i}=\left\lfloor\frac{r_{i}}{t}\right\rfloor$ for $1 \leqslant i \leqslant a$. Let $m=m_{1}+\cdots+m_{a}$. Write $r_{i_{0}}=c_{i_{0}} t+s_{i_{0}}$ for $s_{i_{0}} \in\{0,1\}$ and assume $t \mid r_{i}$ for all $i \neq i_{0}$. We have 


$$
\begin{aligned}
& \lim _{q \rightarrow \zeta^{d}} X(q)=\left(\begin{array}{c}
d \\
m_{1}
\end{array}\right)\left(\begin{array}{c}
d-m_{1} \\
m_{2}
\end{array}\right) \cdots\left(\begin{array}{c}
d-\left(m-m_{a}\right) \\
m_{a}
\end{array}\right) \lim _{q \rightarrow \zeta^{d}} \frac{[b-1-m t] !_{q}\left[r_{i_{0}}-s_{i_{0}}\right] !_{q}}{\left[r_{i_{0}}\right] !_{q}[b-k] !_{q}} \\
& =\left(\begin{array}{c}
d \\
m_{1}, \ldots, m_{a}, d-m
\end{array}\right) \lim _{q \rightarrow \zeta^{d}} \frac{\left[b-1-\left(k-s_{i_{0}}\right)\right] !_{q}\left[r_{i_{0}}-s_{i_{0}}\right] !_{q}}{\left[r_{i_{0}}\right] !_{q}[b-k] !_{q}} b \\
& =\left\{\begin{array}{cc}
\left(\begin{array}{c}
d \\
m_{1}, \ldots, m_{a}, d-m \\
d
\end{array}\right) \lim _{q \rightarrow \zeta^{d}} \frac{1}{[b-k]_{q}} & s_{i_{0}}=0 \\
\left(\begin{array}{c}
d \\
m_{1}, \ldots, m_{a}, d-m
\end{array}\right) \lim _{q \rightarrow \zeta^{d}} \frac{1}{\left[r_{i_{0}}\right]_{q}} & s_{i_{0}}=1
\end{array}\right. \\
& =\left(\begin{array}{c}
d \\
m_{1}, \ldots, m_{a}, d-m
\end{array}\right) \text {. }
\end{aligned}
$$

By Corollary 4.10, we have $X\left(\zeta^{d}\right)=\left|X^{\text {rot }^{d}}\right|$.

The following Narayana version of Theorem 5.1 proves a CSP involving the action of rotation on $a, b$-noncrossing partitions with a fixed number of blocks.

Theorem 5.2. Let $a<b$ be coprime, let $1 \leqslant k \leqslant a$, and let $X$ be the set of $(a, b)$ noncrossing partitions of $[b-1]$ with $k$ blocks.

The triple $(X, C, X(q))$ exhibits the cyclic sieving phenomenon, where $C=\mathbb{Z}_{b-1}$ acts on $X$ by rotation and

$$
X(q)=\operatorname{Nar}_{q}(a, b, k)=\frac{1}{[a]_{q}}\left[\begin{array}{l}
a \\
k
\end{array}\right]_{q}\left[\begin{array}{l}
b-1 \\
k-1
\end{array}\right]_{q}
$$

is the q-rational Narayana number.

Proof. Reiner and Sommers proved that the $q$-Narayana numbers $\operatorname{Nar}_{q}(a, b, k)$ are polynomials in $q$ with nonnegative integer coefficients [9]. As in the Kreweras case, no combinatorial proof of this fact is known.

On the other hand, the polynomiality and nonnegativity of $\operatorname{Nar}_{q}(a, b, k)$ may be proven by a direct argument similar to that in the Kreweras case. By the same reasoning as in the proof of Theorem 5.1, we get that

$$
\operatorname{Nar}_{q}(a, b, k)=\prod_{d \geqslant 2} \Phi_{d}(q)^{f_{d}}
$$

where

$$
\begin{aligned}
f_{d} & =-\chi(d \mid a)+\left(\left\lfloor\frac{a}{d}\right\rfloor-\left\lfloor\frac{k}{d}\right\rfloor-\left\lfloor\frac{a-k}{d}\right\rfloor\right)+\left(\left\lfloor\frac{b-1}{d}\right\rfloor-\left\lfloor\frac{k-1}{d}\right\rfloor-\left\lfloor\frac{b-k}{d}\right\rfloor\right) \\
& =-\chi(d \mid k)+\left(\left\lfloor\frac{a-1}{d}\right\rfloor-\left\lfloor\frac{k-1}{d}\right\rfloor-\left\lfloor\frac{a-k}{d}\right\rfloor\right)+\left(\left\lfloor\frac{b-1}{d}\right\rfloor-\left\lfloor\frac{k-1}{d}\right\rfloor-\left\lfloor\frac{b-k}{d}\right\rfloor\right) .
\end{aligned}
$$

The terms in the parentheses of either of the right-hand sides above are clearly nonnegative. It follows that $f_{d} \geqslant 0$ whenever $d \nmid a$ or $d \nmid k$. 
Suppose $d \mid a$ and $d \mid k$. Since $a$ and $b$ are coprime we conclude that $d \nmid b$ and $\left\lfloor\frac{b-1}{d}\right\rfloor=\left\lfloor\frac{b}{d}\right\rfloor$. This means that the term in the second parentheses of in the right-hand sides of Equations 24 or 25 can be expressed as

$$
\begin{aligned}
\left(\left\lfloor\frac{b-1}{d}\right\rfloor-\left\lfloor\frac{k-1}{d}\right\rfloor-\left\lfloor\frac{b-k}{d}\right\rfloor\right) & =\left(\left\lfloor\frac{b}{d}\right\rfloor-\left\lfloor\frac{k-1}{d}\right\rfloor-\left\lfloor\frac{b-k}{d}\right\rfloor\right) \\
& =\left(\left\lfloor\frac{b}{d}\right\rfloor-\left(\frac{k}{d}-1\right)-\left(\left\lfloor\frac{b}{d}\right\rfloor-\frac{k}{d}\right)\right) \\
& =1
\end{aligned}
$$

where the second equality used the fact that $d \mid k$. We conclude that

$$
f_{d} \geqslant-1+0+1=0
$$

as desired, and conclude that $\operatorname{Nar}_{q}(a, b, k)$ is a polynomial in $q$ with integer coefficients.

To complete the proof that $\operatorname{Nar}_{q}(a, b, k)$ is a polynomial with nonnegative integer coefficients, simply observe that

$$
[a]_{q} \times \operatorname{Nar}_{q}(a, b, k)=\left[\begin{array}{l}
a \\
k
\end{array}\right]_{q}\left[\begin{array}{l}
b-1 \\
k-1
\end{array}\right]_{q}
$$

is a product of two $q$-binomial coefficients, and hence a unimodal and reciprocal polynomial in $q$ with nonnegative coefficients. By [1, Proposition 10.1 (iii)] and the fact that $\operatorname{Nar}_{q}(a, b, k)$ is a polynomial with integer coefficients, we get that the coefficients of $\operatorname{Nar}_{q}(a, b, k)$ are nonnegative.

Let $\zeta=e^{\frac{2 \pi i}{b-1}}$ and let $d \mid(b-1)$ with $1 \leqslant d<b-1$. Let $q=\frac{b-1}{d}$. By Corollary 4.11, it is enough to show that

$$
X\left(\zeta^{d}\right)= \begin{cases}\left(\begin{array}{c}
d \\
\left\lfloor\frac{k}{d}\right\rfloor
\end{array}\right)\left(\begin{array}{c}
\left\lfloor\frac{a d}{b-1}\right\rfloor-1 \\
\left\lfloor\frac{k}{q}\right\rfloor-1
\end{array}\right) & \text { if } k \equiv 0(\bmod q), \\
\left(\begin{array}{c}
d \\
\left\lfloor\frac{k}{q}\right\rfloor
\end{array}\right)\left(\begin{array}{c}
\left\lfloor\frac{a d}{b-1}\right\rfloor \\
\left\lfloor\frac{k}{q}\right\rfloor
\end{array}\right) & \text { if } k \equiv 1(\bmod q), \\
0 & \text { otherwise. }\end{cases}
$$

The argument here is similar to that in the proof of Theorem 5.1 and is left to the reader.

The next CSP was asked for in [4, Subsection 6.2].

Theorem 5.3. Let $a<b$ be coprime and let $X$ be the set of $(a, b)$-noncrossing partitions of $[b-1]$.

The triple $(X, C, X(q))$ exhibits the cyclic sieving phenomenon, where $C=\mathbb{Z}_{b-1}$ acts on $X$ by rotation and

$$
X(q)=\operatorname{Cat}_{q}(a, b)=\frac{1}{[a+b]_{q}}\left[\begin{array}{c}
a+b \\
a, b
\end{array}\right]_{q}
$$

is the q-rational Catalan number. 
Proof. It can be argued directly that $\mathrm{Cat}_{q}(a, b)$ is a polynomial in $q$ with nonnegative integer coefficients using methods similar to those appearing in the proofs of Theorems 5.1 and 5.2. One shows that we have

$$
\operatorname{Cat}_{q}(a, b)=\prod_{d \geqslant 2} \Phi_{d}(q)^{h_{d}}
$$

where

$$
\begin{aligned}
h_{d} & =-\chi(d \mid(a+b))+\left(\left\lfloor\frac{a+b}{d}\right\rfloor-\left\lfloor\frac{a}{d}\right\rfloor-\left\lfloor\frac{b}{d}\right\rfloor\right) \\
& =-\chi(d \mid a)+\left(\left\lfloor\frac{a+b-1}{d}\right\rfloor-\left\lfloor\frac{a-1}{d}\right\rfloor-\left\lfloor\frac{b}{d}\right\rfloor\right) .
\end{aligned}
$$

Since the terms in the parentheses in the right-hand sides of Equations 29 and 30 are both nonnegative, we conclude that $h_{d} \geqslant 0$ unless $d \mid(a+b)$ and $d \mid a$. Since $a$ and $b$ are coprime, these latter divisibilities cannot hold simultaneously and we conclude that $\mathrm{Cat}_{q}(a, b) \in \mathbb{Z}[q]$. Since

$$
[a+b]_{q} \times \operatorname{Cat}_{q}(a, b)=\left[\begin{array}{c}
a+b \\
a, b
\end{array}\right]_{q}
$$

is a unimodal and reciprocal polynomial in $q$ with nonnegative integer coefficients, we conclude as before that $\operatorname{Cat}_{q}(a, b)$ has nonnegative coefficients.

Let $\zeta=e^{\frac{2 \pi i}{b-1}}$ and let $d \mid(b-1)$ with $1 \leqslant d<b-1$. By Corollary 4.12 it is enough to show that

$$
X(q)=\left(\begin{array}{c}
\left\lfloor\frac{a d}{b-1}\right\rfloor+d \\
d
\end{array}\right)
$$

The argument here is similar to that in the proof of Theorem 5.1 and is left to the reader.

Our final CSP proves [4, Conjecture 5.3].

Theorem 5.4. Let $(a, b)$ be coprime and $X$ be the set of homogeneous $(a, b)$-noncrossing partitions of $[a+b-1]$. The triple $(X, C, X(q))$ exhibits the cyclic sieving phenomenon, where $C=\mathbb{Z}_{a+b-1}$ acts on $X$ by rotation and

$$
X(q)=\operatorname{Cat}_{q}(a, b)=\frac{1}{[a+b]_{q}}\left[\begin{array}{c}
a+b \\
a, b
\end{array}\right]_{q}
$$

is the q-rational Catalan number.

Proof. Let $D$ be an $a, b$-Dyck path. Construct an $a, a+b$-Dyck path $D^{\prime}$ by replacing every north step in $D$ with a pair $N E$. Then $D^{\prime}$ has $a$ vertical runs and $\pi(D)=\pi\left(D^{\prime}\right)$. Moreover, the map $D \mapsto D^{\prime}$ gives a bijection $\{$ all $a, b$-Dyck paths $\} \longrightarrow\{$ all $a, a+b$-Dyck paths with $a$ vertical runs $\}$.

It follows that the set $\operatorname{HNC}(a, b)$ of homogeneous $a, b$-noncrossing partitions of $[a+b-1]$ is precisely the set of (ordinary) $a, a+b$-noncrossing partitions of $[a+b-1]$ with precisely $a$ blocks. The result follows from Theorem 5.2. 


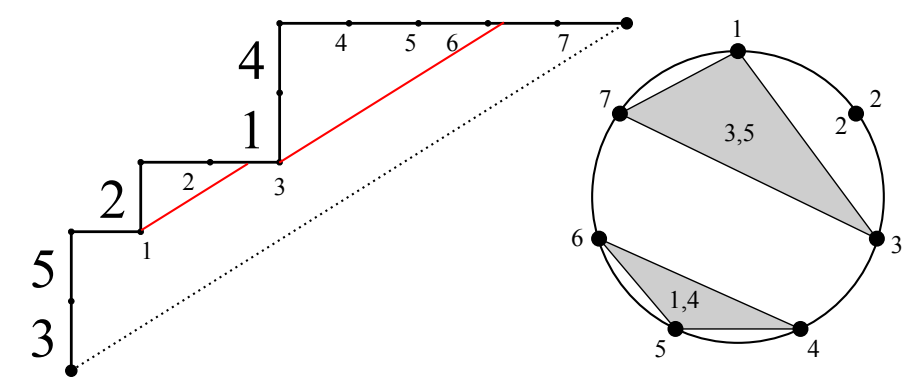

Figure 5: A 5, 8-noncrossing parking function.

\section{Parking functions}

\subsection{Noncrossing parking functions}

Let $W$ be an irreducible real reflection group with Coxeter number $h$. Armstrong, Reiner, and Rhoades defined a $W \times \mathbb{Z}_{h}$-set Park $k_{W}^{N C}$ called the set of $W$-noncrossing parking functions [3]. Given a Fuss parameter $m \geqslant 1$, a Fuss extension $\operatorname{Park}_{W}^{N C}(m)$ of Park $\operatorname{Pa}_{W}^{N C}$ was defined in [12]. An increasingly strong trio of conjectures (Weak, Intermediate, and Strong) was formulated about these objects and it was shown that the weakest of these uniformly implies various facts from $W$-Catalan theory which are at present only understood in a case-by-case fashion.

In this section, we give a rational extension of the constructions in $[3,12]$ when $W=\mathfrak{S}_{a}$ is the symmetric group. This gives evidence that $\mathrm{NC}(a, b)$ gives the 'correct' definition of rational noncrossing partitions. Extending the work of $[3,12]$ to other reflection groups remains an open problem.

Definition 6.1. Let $a<b$ be coprime. An $a, b$-noncrossing parking function is a pair $(\pi, f)$ where $\pi \in \mathrm{NC}(a, b)$ is an a,b-noncrossing partition and $B \mapsto f(B)$ is a labeling of the blocks of $\pi$ with subsets of $[a]$ such that

- we have $[a]=\biguplus_{B \in \pi} f(B)$, and

- for all blocks $B \in \pi$ we have $|f(B)|=\operatorname{rank}_{a, b}^{\pi}(B)$.

We denote by $\operatorname{Park}^{N C}(a, b)$ the set of all $a, b$-noncrossing parking functions.

An example of a 5, 8-noncrossing parking function is shown in Figure 5. The parking function shown is $(\pi, f)$, where $\pi=\{\{1,3,7\},\{2\},\{4,5,6\}\}$ and $f$ is the labeling $\{1,3,7\} \mapsto\{3,5\},\{4,5,6\} \mapsto\{1,4\}$, and $\{2\} \mapsto\{2\}$.

By Proposition 3.6, when $(a, b)=(n, m n+1)$, the set $\operatorname{Park}^{N C}(n, m n+1)$ agrees with the construction of $\operatorname{Park}_{\mathfrak{S}_{n}}^{N C}(m)$ given in [12]. In the classical case $(a, b)=(n, n+1)$, the set $\operatorname{Park}^{N C}(n, n+1)$ appeared in the work of Edelman under the name of '2-noncrossing partitions' [7]. The set Park ${ }^{N C}(a, b)$ carries an action of $\mathfrak{S}_{a} \times \mathbb{Z}_{b-1}$.

Proposition 6.2. The set $\operatorname{Park}^{N C}(a, b)$ carries an action of the product group $\mathfrak{S}_{a} \times \mathbb{Z}_{b-1}$, where $\mathfrak{S}_{a}$ acts by label permutation and $\mathbb{Z}_{b-1}$ acts by rotation. 
Proof. We need to know that rotation preserves $a$, $b$-ranks of blocks, which is Proposition 3.10 .

In order to state a formula for the character of the action in Proposition 6.2, we will need some notation. Let $V=\mathbb{C}^{a} /\langle(1, \ldots, 1)\rangle$ be the reflection representation of $\mathfrak{S}_{a}$ and let $\zeta=e^{\frac{2 \pi i}{b-1}}$. Given $w \in \mathfrak{S}_{a}$ and $d \geqslant 0$, let $\operatorname{mult}_{w}\left(\zeta^{d}\right)$ be the multiplicity of $\zeta^{d}$ as an eigenvalue in the action of $w$ on $V$. With this notation, a formula for the character $\chi$ is given by the following formula.

Theorem 6.3. Let $w \in \mathfrak{S}_{a}$ and let $g$ be a generator of $\mathbb{Z}_{b-1}$. We have

$$
\chi\left(w, g^{d}\right)=b^{\text {mult }_{w}\left(\zeta^{d}\right)}
$$

for all $w \in \mathfrak{S}_{a}$ and $d \geqslant 0$.

The multiplicity $\operatorname{mult}_{w}\left(\zeta^{d}\right)$ can be read off from the cycle structure of $w$. Namely, for $d \mid b-1$ we have

$$
\operatorname{mult}_{w}\left(\zeta^{d}\right)= \begin{cases}\#(\text { cycles of } w)-1 & \text { if } q=1 \\ \#(\text { cycles of } w \text { of length divisible by } q) & \text { otherwise }\end{cases}
$$

where $q=\frac{b-1}{d}$.

When $(a, b)=(n, n+1)$, Proposition 6.3 was proven in [3, Section 8]. When $(a, b)=$ $(n, m n+1)$, Proposition 6.3 is [12, Proposition 8.6]. The character formula of Equation 33 is a rational extension of the Weak Conjecture of $[3,12]$ when $W=\mathfrak{S}_{a}$ is the symmetric group.

The argument used to prove [12, Proposition 8.6] can be combined with the enumerative results of Section 4 to prove Theorem 6.3. We quickly illustrate how this is done.

Proof. (of Theorem 6.3) Let $\left(w, g^{d}\right) \in \mathfrak{S}_{a} \times \mathbb{Z}_{b-1}$. We want to show that $\chi\left(w, g^{d}\right)=$ $b^{\text {mult }_{w}\left(\zeta^{d}\right)}$. Without loss of generality, we may assume that $d \mid(b-1)$. Let $q:=\frac{b-1}{d}$. The argument depends on whether $q=1$ or $q>1$.

Case 1: $q=1$. In this case, we are ignoring the action of $\mathbb{Z}_{b-1}$ and considering $\operatorname{Park}^{N C}(a, b)$ as an $\mathfrak{S}_{a}$-set. We construct an $\mathfrak{S}_{a}$-equivariant bijection from Park ${ }^{N C}(a, b)$ to another $\mathfrak{S}_{a}$-set which is known to have the correct character.

Let Park Pabb $_{a}$ be the set of all sequences $\left(p_{1}, p_{2}, \ldots, p_{a}\right)$ of positive integers whose nondecreasing rearrangement $\left(p_{1}^{\prime} \leqslant p_{2}^{\prime} \leqslant \cdots \leqslant p_{a}^{\prime}\right)$ satisfies $p_{i}^{\prime} \leqslant \frac{b}{a}(i-1)+1$. Equivalently, the histogram with left-to-right heights $\left(p_{1}^{\prime}-1, p_{2}^{\prime}-1, \ldots, p_{a}^{\prime}-1\right)$ stays below the line $y=\frac{b}{a} x$. Sequences in Park $a, b$ are called rational slope parking functions.

The symmetric group $\mathfrak{S}_{a}$ acts on Park $\operatorname{Pa}_{a, b}$ by $w \cdot\left(p_{1}, p_{2}, \ldots, p_{a}\right):=\left(p_{w(1)}, p_{w(2)}, \ldots, p_{w(a)}\right)$. It is known that the character of this action is given by Equation 33 with $\zeta=1$.

We build an $\mathfrak{S}_{a}$-equivariant bijection $\varphi: \operatorname{Park}^{N C}(a, b) \stackrel{\sim}{\rightarrow} \operatorname{Park}_{a, b}$ as follows. Let $(\pi, f)$ be an $a, b$-noncrossing parking function. Define a sequence $\left(p_{1}, p_{2}, \ldots, p_{a}\right)$ by letting $p_{i}=\min (B)$, where $B$ is the unique block of $\pi$ satisfying $i \in f(B)$. Proposition 3.8

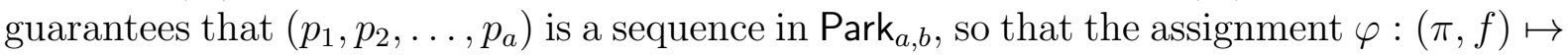


$\left(p_{1}, p_{2}, \ldots, p_{a}\right)$ gives a well defined function $\varphi: \operatorname{Park}^{N C}(a, b) \rightarrow \operatorname{Park}_{a, b}$. It is clear that $\varphi$ is $\mathfrak{S}_{a}$-equivariant. Moreover, if $\varphi(\pi, f)=\left(p_{1}, p_{2}, \ldots, p_{a}\right)$, then we can recover both the minimal elements of the blocks of $\pi$ (these are the entries appearing in $p_{1}, p_{2}, \ldots, p_{a}$ ) and the ranks of these blocks (the rank of a block $B$ is the number of times $\min (B)$ occurs in $\left.p_{1}, p_{2}, \ldots, p_{a}\right)$. Proposition 3.8 says that the $a, b$-noncrossing partition $\pi$ is therefore determined from $\left(p_{1}, p_{2}, \ldots, p_{a}\right)$. It is easy to see that we can determine the entire $a, b$ noncrossing function $(\pi, f)$, so that $\varphi$ is an $\mathfrak{S}_{a}$-equivariant bijection.

Case 2: $q>1$. This argument is a rational extension of [12, Section 8]. Let $r_{q}(w)$ be the number of cycles of $w$ having length divisible by $q$. We need to show that

$$
\left|\operatorname{Park}^{N C}(a, b)^{\left(w, g^{d}\right)}\right|=b^{r_{q}(w)},
$$

where $\operatorname{Park}^{N C}(a, b)^{\left(w, g^{d}\right)}$ is the set of $a, b$-noncrossing parking functions fixed by $\left(w, g^{d}\right)$. The idea is to show that both sides of Equation 35 count a certain set of functions.

Let $g$ act on the set $[b-1] \cup\{0\}$ by the permutation $(1,2, \ldots, b-1)(0)$. A function $e:[a] \rightarrow[b-1] \cup\{0\}$ is said to be $\left(w, g^{d}\right)$-equivariant if we have

$$
e(w(j))=g^{d} e(j)
$$

for every $1 \leqslant j \leqslant a$. We claim that the number of $\left(w, g^{d}\right)$-equivariant functions $e:[a] \rightarrow$ $[b-1] \cup\{0\}$ is equal to $b^{r_{q}(w)}$. Indeed, the values of $e$ on any cycle of $w$ are determined by the value of $e$ on any representative of that cycle. Moreover, unless a cycle of $w$ has length divisible by $q$, the relation $e(w(j))=g^{d} e(j)$ forces $e(j)=0$ for all $j$ belonging to that cycle. For every cycle of $w$ having length divisible by $q$, we have $b$ choices for $e(j)$, where $j$ is a chosen cycle representative.

By considering set partitions coming from preimages, we can get another expression for the number of $\left(w, g^{d}\right)$-admissible functions $[a] \rightarrow[b-1] \cup\{0\}$. A set partition $\sigma=$ $\left\{B_{1}, B_{2}, \ldots\right\}$ of $[a]$ is called $(w, q)$-admissible if

- $\sigma$ is $w$-stable in the sense that $w(\sigma)=\left\{w\left(B_{1}\right), w\left(B_{2}\right), \ldots\right\}=\sigma$,

- at most one block $B_{i_{0}}$ of $\sigma$ is itself $w$-stable in the sense that $w\left(B_{i_{0}}\right)=B_{i_{0}}$, and

- for any block $B_{i}$ of $\sigma$ which is not $w$-stable, the blocks

$$
B_{i}, w\left(B_{i}\right), w^{2}\left(B_{i}\right), \ldots, w^{q-1}\left(B_{i}\right)
$$

are pairwise distinct, and $w^{q}\left(B_{i}\right)=B_{i}$.

It is straightforward to see that, for any $\left(w, g^{d}\right)$-equivariant function $e:[a] \rightarrow[b-1] \cup\{0\}$, the set partition $\sigma$ of $[a]$ defined by $i \sim j$ if and only if $e(i)=e(j)$ is $(w, q)$-admissible. On the other hand, the same argument as in [12, Proof of Lemma 8.4] shows that the number of $\left(w, g^{d}\right)$-equivariant functions $e:[a] \rightarrow[b-1] \cup\{0\}$ which induce a fixed $(w, q)$ admissible set partition $\sigma$ of $[a]$ is $(b-1)(b-1-q)(b-1-2 q) \cdots\left(b-1-\left(t_{\sigma}-1\right) q\right)$, where $t_{\sigma}$ is the number of non-singleton $w$-orbits of blocks of $\sigma$. Combining this with the last paragraph, we get that

$$
b^{r_{q}(w)}=\sum_{\sigma}(b-1)(b-1-q)(b-1-2 q) \cdots\left(b-1-\left(t_{\sigma}-1\right) q\right),
$$


where the sum is over all $(w, q)$-admissible set partitions $\sigma$ of $[a]$.

To relate Equation 36 to parking functions, for $(\pi, f) \in \operatorname{Park}^{N C}(a, b)$ we let $\tau(\pi, f)$ be the set partition of $[a]$ defined by $i \sim j$ if and only if $i, j \in f(B)$ for some block $B \in \pi$. If $(\pi, f) \in \operatorname{Park}^{N C}(a, b)^{\left(w, g^{d}\right)}$, it follows that $\tau(\pi, f)$ is a $(w, q)$-admissible set partition of $[a]$. On the other hand, if $\sigma$ is a fixed $(w, q)$-admissible partition of $[a]$, we claim that the number of parking functions $(\pi, f) \in \operatorname{Park}^{N C}(a, b)^{\left(w, g^{d}\right)}$ with $\tau(\pi, f)=\sigma$ equals $(b-1)(b-1-q)(b-1-2 q) \cdots\left(b-1-\left(t_{\sigma}-1\right) q\right)$, where $t_{\sigma}$ is the number of nonsingleton $w$-orbits of blocks in $\sigma$.

To see why this claim is true, we consider how to construct an $a, b$-noncrossing parking function $(\pi, f) \in \operatorname{Park}^{N C}(a, b)^{\left(w, g^{d}\right)}$ with $\tau(\pi, f)=\sigma$. This argument is almost the same as that proving [12, Lemma 8.5], but it will rely on Corollary 4.10.

We first construct an $a, b$-noncrossing partition $\pi$ which is invariant under $\operatorname{rot}^{d}$. If $\sigma$ has $m_{i}$ non singleton $w$-orbits of blocks of size $i$ for $1 \leqslant i \leqslant a$, then $\pi$ must have $m_{i}$ $\left\langle\operatorname{rot}^{d}\right\rangle$-orbits of non-central blocks of rank $i$ for $1 \leqslant i \leqslant a$. Corollary 4.10 says that the number of such partitions $\pi$ is the multinomial coefficient $\left(\begin{array}{c}d \\ m_{1}, m_{2}, \ldots, m_{a}, d-t_{\sigma}\end{array}\right)$. With $\pi$ fixed, we consider how to build the labeling $f$ of the blocks of $\pi$. The labeling $f$ must pair off the $\left\langle\operatorname{rot}^{d}\right\rangle$-orbits of non-central blocks of $\pi$ of rank $i$ with the non-singleton $w$-orbits of blocks of $\sigma$ of size $i$. For every $i$, there are $m_{i}$ ! ways to do this matching. As each of these orbits has size $q$, we also have $q$ ways to rotate $f$ within each orbit after this matching is chosen. In summary, the number of pairs $(\pi, f) \in \operatorname{Park}^{N C}(a, b)^{\left(g, w^{d}\right)}$ satisfying $\tau(\pi, f)=\sigma$ is

$$
\begin{aligned}
& q^{m_{1}} \cdots q^{m_{a}} m_{1} ! \cdots m_{a} !\left(\begin{array}{c}
d \\
m_{1}, \ldots, m_{a}, d-t_{\sigma}
\end{array}\right)=q^{m_{1}} \cdots q^{m_{a}} \frac{d !}{\left(d-t_{\sigma}\right) !} \\
& =(b-1)(b-1-q)(b-1-2 q) \cdots\left(b-1-\left(t_{\sigma}-1\right) q\right) .
\end{aligned}
$$

Applying Equation 36, we obtain Equation 35, completing the proof.

Theorem 6.3 can be strengthened to prove a rational analog of the Generic Strong Conjecture of [13] in type A. Let $V$ be the (complexified) reflection representation of $\mathfrak{S}_{a}$, let $\mathbb{C}[V]=\bigoplus_{d \geqslant 0} \mathbb{C}[V]_{d}$ be its polynomial ring, and equip $\mathbb{C}[V]$ with the graded action of $\mathfrak{S}_{a} \times \mathbb{Z}_{b-1}$ given by letting $\mathfrak{S}_{a}$ act by linear substitutions and the generator $g$ of $\mathbb{Z}_{b-1}$ scale by $\left(e^{\frac{2 \pi i}{b-1}}\right)^{d}$ in degree $d$. We identify $\mathbb{C}[V]_{1}$ with the dual space $V^{*}$ and consider the set of $\mathbb{C}\left[\mathfrak{S}_{a}\right]$-equivariant linear maps $\operatorname{Hom}_{\mathbb{C}\left[\mathfrak{S}_{a}\right]}\left(V^{*}, \mathbb{C}[V]_{b}\right)$ as an affine complex space. We refer the reader to [13] for the definitions of the objects in the following result.

Theorem 6.4. Let $\mathcal{R} \subset \operatorname{Hom}_{\mathbb{C}\left[\mathfrak{S}_{a}\right]}\left(V^{*}, \mathbb{C}[V]_{b}\right)$ be the set of $\Theta \in \operatorname{Hom}_{\mathbb{C}\left[\mathfrak{S}_{a}\right]}\left(V^{*}, \mathbb{C}[V]_{b}\right)$ such that the 'parking locus' $V^{\Theta}(b) \subset V$ cut out by the ideal $\left\langle\Theta\left(x_{1}\right)-x_{1}, \ldots, \Theta\left(x_{a-1}\right)-x_{a-1}\right\rangle \subset$ $\mathbb{C}[V]$ is reduced (here $x_{1}, \ldots, x_{a-1}$ is any basis of $V^{*}$ ). For any $\Theta \in \mathcal{R}$, there exists an equivariant bijection of $\mathfrak{S}_{a} \times \mathbb{Z}_{b-1}$-sets

$$
V^{\Theta}(b) \cong \mathfrak{S}_{a} \times \mathbb{Z}_{b-1} \operatorname{Park}^{N C}(a, b) .
$$

Moreover, there exists a nonempty Zariski open subset $\mathcal{U} \subseteq \operatorname{Hom}_{\mathbb{C}_{\left[\mathfrak{S}_{a}\right]}}\left(V^{*}, \mathbb{C}[V]_{b}\right)$ such that $\mathcal{U} \subseteq \mathcal{R}$. 
The proof of Theorem 6.4 is almost a word-for-word recreation of [13, Sections 4, 5]. One need only replace the reference to the proof of [12, Lemma 8.5] in the proof of [13, Lemma 4.6] with the corresponding argument the fifth paragraph of Case 2 in the proof of Theorem 6.3 (which ultimately relies on Corollary 4.10).

\section{Closing Remarks}

The research program of Coxeter-Catalan combinatorics seeks to realize Catalan objects as the symmetric group instances of a wider class of objects one can attach to a broader class of reflection groups $W$. Reiner [8] described a $W$-analog of a noncrossing partition for classical reflection groups $W$ which reduces to the usual notion of a noncrossing partition of $[n]$ when $W=\mathfrak{S}_{n}$ (see also [5,6]). In his Ph.D. thesis, Armstrong [2] studied a FussCatalan generalization of $W$-noncrossing partitions which depends on a reflection group $W$ and a Fuss parameter $m \in \mathbb{Z}_{>0}$.

It is natural to ask for a notion of a ' $W$ - $b$-rational noncrossing partition', defined for any reflection group $W$ and depending on a 'rational parameter' $b \in \mathbb{Z}_{>0}$ which is coprime to the Coxeter number of $W$. The set $\mathrm{NC}(W, b)$ of $W$-b-rational noncrossing partitions should reduce to $\mathrm{NC}(a, b)$ when $W=\mathfrak{S}_{a}$. Moreover, the favorable combinatorial properties of $\mathrm{NC}(a, b)$ (such as a dihedral action with a natural cyclic sieving phenomenon) should be natural specializations of more general features of $\mathrm{NC}(W, b)$.

The problem of extending rational Catalan combinatorics to other reflection groups $W$ remains almost entirely open. In particular, no $W$-analog of the rational noncrossing partitions is known. However, the results of this paper give a candidate for rational noncrossing partitions associated to the hyperoctohedral group.

Let $W\left(B_{n}\right)$ denote the hyperoctohedral group of signed permutations of $[n]$. In the classical and Fuss-Catalan cases, objects associated to the group $W\left(B_{n}\right)$ are obtained by considering those attached to the 'doubled' symmetric group $\mathfrak{S}_{2 n}$ which are invariant under antipodal symmetry (see [8]). When $(a, d) \rightarrow\left(2 n, \frac{b-1}{2}\right)$, the formulas in Corollaries $4.10,4.11$, and 4.12 reduce to the hyperoctohedral analogs of the rational Kreweras, Narayana, and Catalan numbers (here we view $2 n$ as the Coxeter number of $W\left(B_{n}\right)$ and let the rational parameter $b$ be coprime to $2 n$ ). Thus, restricting to objects with antipodal symmetry gives the correct numerology for type $\mathrm{B}$, even in the rational setting.

This paper has focused completely on the combinatorics of $\mathrm{NC}(a, b)$; the symmetric group $\mathfrak{S}_{a}$ made no appearance. However, we are hopeful that the combinatorial results and techniques in this paper will prove useful in defining and studying $W$-rational noncrossing partitions for arbitrary reflection groups $W$.

\section{Acknowledgements}

The authors are grateful to Drew Armstrong and Vic Reiner for helpful conversations. The authors are also indebted to an extremely diligent and helpful referee who allowed us to reproduce their direct proof of the polynomiality and nonnegativity of the $q$-rational 
Kreweras polynomials (as well as alerting us to similar direct proofs of the polynomiality and nonnegativity in the Narayana and Catalan cases). B. Rhoades was partially supported by NSF grants DMS - 1068861 and DMS - 1500838.

\section{References}

[1] G. E. Andrews, The Friedman-Joichi-Stanton monotonicity conjecture at primes, Unusual Approaches of Number Theory (M. Nathanson, ed.), DIMACS Ser. Discrete Math. Theor. Comp. Sci., vol. 64, Amer. Math. Soc., Providence, RI, 2004, pp. 9-15.

[2] D. Armstrong. Generalized noncrossing partitions and the combinatorics of Coxeter groups. Mem. Amer. Math. Soc., (2009), no. 949, Amer. Math. Soc., Providence, RI.

[3] D. Armstrong, V. Reiner, and B. Rhoades. Parking spaces. Adv. Math., 269 (2015), 647-706.

[4] D. Armstrong, B. Rhoades, and N. Williams. Rational associahedra and noncrossing partitions. Electron. J. Combin., 20 (3) (2013), \#P54.

[5] C. Athanasiadis. On noncrossing and nonnesting partitions for the classical groups. Electron. J. Combin., 5 (1998), \#R42.

[6] D. Bessis and V. Reiner. Cyclic sieving of noncrossing partitions for complex reflection groups. Ann. Comb., 15 (2011), 197-222.

[7] P. Edelman. Chain enumeration and non-crossing partitions. Discrete Math., 31 (1980), 171-180.

[8] V. Reiner. Non-crossing partitions for classical reflection groups. Discrete Math., 177 (1997), 195-222.

[9] V. Reiner and E. Sommers. $q$-Narayana and $q$-Kreweras number for Weyl groups. In preparation, 2016. Slides available at http://www.math.umn.edu/ reiner/Talks/ WachsFest.pdf.

[10] V. Reiner, D. Stanton, and D. White. The cyclic sieving phenomenon. J. Comb. Theory, Ser. A, 108 (2004), 17-50.

[11] B. Rhoades. Alexander duality and rational associahedra. SIAM J. Discrete Math., 29 (1) (2015), 431-460.

[12] B. Rhoades. Parking structures: Fuss analogs. J. Algebraic Combin., 40 (2014), 417473.

[13] B. Rhoades. Evidence for parking conjectures. Submitted, 2015. arXiv: 1504.06869. 